TRANSACTIONS OF THE

AMERICAN MATHEMATICAL SOCIETY

Volume 285, Number 1, September 1984

\title{
CYCLIC VECTORS IN THE DIRICHLET SPACE
}

BY

\author{
LEON BROWN AND ALLEN L. SHIELDS ${ }^{1}$
}

\begin{abstract}
We study the Hilbert space of analytic functions with finite Dirichlet integral in the open unit disc. We try to identify the functions whose polynomial multiples are dense in this space. Theorems 1 and 2 confirm a special case of the following conjecture: if $|f(z)| \geqslant|g(z)|$ at all points and if $g$ is cyclic, then $f$ is cyclic. Theorems 3-5 give a sufficient condition ( $f$ is an outer function with some smoothness and the boundary zero set is at most countable) and a necessary condition (the radial limit can vanish only for a set of logarithmic capacity zero) for a function $f$ to be cyclic.
\end{abstract}

Introduction. In this paper we shall study the (Hilbert) space of analytic functions in the open unit disc $\Delta$ in the complex plane that have a finite Dirichlet integral: $\iint\left|f^{\prime}\right|^{2} d x d y<\infty$. Our goal is to identify, as far as possible, the "cyclic vectors" in this space, that is, those functions $f$ such that the polynomial multiples of $f$ are dense in the space. The corresponding problem for the Hardy space $H^{2}$ was solved by Beurling [5] in 1949: the cyclic vectors are precisely the outer functions. The present paper is divided into four sections; it contains 20 propositions, 5 theorems and 19 unsolved problems (stated as Questions in the text).

The first section deals with cyclic vectors and multiplication operators in a general Banach space of analytic functions (in a bounded region of the complex plane). The theory is illustrated by considering a special family of Hilbert spaces, denoted $\left\{D_{\alpha}\right\}$, $-\infty<\alpha<\infty$, in the unit disc. (The values $\alpha=0,1$ give, respectively, $H^{2}$ and the Dirichlet space.) These spaces (for $0<\alpha \leqslant 1$ ) were considered by Carleson in his dissertation [7]. This section contains 10 propositions and raises 6 questions, mostly for a general Banach space of analytic functions. For example, Question 3 asks if $f$ must be cyclic whenever we have $|f(z)| \geqslant|g(z)|$ for some cyclic $g$, and all $z$ ? Question 4 asks if $f$ must be cyclic whenever $f$ and $1 / f$ are both in the space. No examples are known where either of these questions has a negative answer.

In $\$ 2$ we begin the study of cyclic vectors in the Dirichlet space $D$. Theorems 1 and 2 give a partial answer to Question 3 above, for this space. This section also contains 2 propositions $(10,11)$ and 4 questions (7-10). Proposition 11 says that if $f$ and $g$ are bounded functions in $D$ whose product is cyclic, then both $f$ and $g$ must be cyclic. Theorem 2 gives a partial converse (we require that $|g|$ be Dini continuous on

Received by the editors July 21, 1983.

1980 Mathematics Subject Classification. Primary 30H05; Secondary 46E15, 46E20, 47B37.

Key words and phrases. Banach spaces of analytic functions, Dirichlet integral, weak invertibility, linear operator, invariant subspace, logarithmic capacity.

${ }^{1}$ The research of both authors was supported in part by the National Science Foundation. 
the boundary of the disc). Question 9 asks if $f$ must be cyclic whenever $f, 1 / f \in D$ and $f$ is bounded.

$\$ 3$ we give examples of different classes of cyclic vectors in $D$. For example, if $f$ is analytic on the closed unit disc and has no zeros in the open disc, then $f^{\alpha}$ is cyclic for all (complex) $\alpha$ having positive real part. Theorems 3 and 4 say that if an outer function $f$ has some additional smoothness (specifically, $f^{\prime} \in H^{2}$ ) and if the boundary zero set is at most countable, then $f$ is cyclic in $D$. This answers a question raised by N. U. Arakelian. In contrast to this, Theorem 5 shows that if $f$ is any function in $D$ whose radial limit function vanishes on a boundary set of positive logarithmic capacity, then $f$ is not cyclic. This section also contains 5 propositions (13-17) and raises 4 questions (11-14). For example, Question 12: is the converse to Theorem 5 valid for outer functiions? An affirmative answer would yield the following characterization: $f \in D$ is cyclic if and only if $f$ is an outer function whose radial limits are zero only for a set of capacity zero. This would imply affirmative answers to Questions 3 and 4 for the space $D$. Question 14: if $f \in D$ is a nonvanishing univalent function, must $f$ be cyclic? An affirmative answer would provide examples of cyclic vectors that are continuous on the closed disc, whose boundary zero set is uncountable.

In $\S 4$ we present some remarks about the independence of Axiom 7 (for a general Banach space of analytic functions) from the remaining axioms; Axiom 7 deals with multiplication operators on the space. Also, we prove three propositions (18-20), dealing with multiplication operators on $D$. For example, Proposition 20: if $\phi \in D$ has a power series with Hadamard gaps, then $\phi$ satisfies a Lipschitz condition of order $\frac{1}{2}$ and $\phi$ is a multiplier on $D$. In addition, 5 questions (15-19) are raised. For example, Question 16: is every $f \in D$ the quotient of two multipliers? (This is true for $H^{2}$ but not for the Bergman space.) Question 17: if $\phi \in D$ satisfies a Lipschitz condition of some positive order, must $\phi$ be a multiplier on $D$ ?

1. Banach spaces of analytic functions. Although we shall mainly be interested in one particular space, we begin by describing a more general situation in which the main problems can be formulated. Let $G$ be a bounded region in the complex plane. We shall say that $E$ is a Banach space of analytic functions on $G$ if the following axioms are satisfied.

1. $E$ is a vector subspace of the space of all holomorphic functions in $G$.

2. $E$ has a norm with respect to which it is complete.

3. The linear functionals of evaluation at a point are continuous with respect to the norm of $E$, for each point in $G$.

4. $E$ contains the polynomials as a dense subset.

5. If $f \in E$, then $z f \in E$.

6. To each point $w \in \partial G$ (the boundary of $G$ ) there corresponds a function $f$ in $E$ that has a singularity at $w$.

We never use this last axiom explicitly, but without it two of the questions that we pose would be trivial. 
Before discussing some consequences of these axioms we turn to a class of examples. Let $\Delta$ denote the open unit disc. By $D_{\alpha}(-\infty<\alpha<\infty)$ we denote the space of functions $f(z)=\Sigma \hat{f}(n) z^{n}$, holomorphic in $\Delta$, for which

$$
\|f\|_{\alpha}^{2}=\sum_{0}^{\infty}(n+1)^{\alpha}|\hat{f}(n)|^{2}<\infty .
$$

For $\alpha<0$ this norm is equivalent to

$$
\iint_{\Delta}\left|f\left(r e^{i \theta}\right)\right|^{2}\left(1-r^{2}\right)^{-1-\alpha} r d r d \theta
$$

(see, for example, [38, Lemma 2]). It is easy to see that $f \in D_{\alpha}$ if and only if $f^{\prime} \in D_{\alpha-2}$. Also, it is not difficult to show that the spaces $D_{\alpha}$ satisfy the six axioms above. (Axiom 3 is proved by applying the Cauchy inequality to the power series for $f(z)$ (see Lemma 1 of [38])). Spaces closely related to the $D_{\alpha}$ spaces $(0<\alpha \leqslant 1)$ have been considered by Carleson in his dissertation [7, \$9], and by Salem and Zygmund [30].

We call attention to the three values of $\alpha:-1,0,1$. The space $D_{-1}$ is called the Bergman space and will be denoted by $B$. The space $D_{0}$ is the Hardy space $H^{2}$. The space $D_{1}$ is called the Dirichlet space and will be denoted by $D$. We have

$$
\begin{aligned}
& \|f\|_{B}^{2}=\sum \frac{|\hat{f}(n)|^{2}}{n+1}=\frac{1}{\pi} \iint\left|f\left(r e^{i \theta}\right)\right|^{2} r d r d \theta, \\
& \|f\|_{D}^{2}=\sum(n+1)|\hat{f}(n)|^{2}=\|f\|_{H^{2}}^{2}+\left\|f^{\prime}\right\|_{B}^{2} .
\end{aligned}
$$

We shall be mainly concerned with the space $D$. This is the space of all those analytic functions that map the unit disk onto a Riemann surface of finite area, the area being equal to $\pi^{-1} \iint\left|f^{\prime}\right|^{2}$. Also, $f \in D$ if and only if $f^{\prime} \in B$.

We return now to our general situation. As before, $E$ will denote a Banach space of analytic functions on a region $G$. Recall that a family of analytic functions in $G$ is said to be a normal family if each sequence in the family contains a subsequence that converges uniformly on each compact subset of $G$. The necessary and sufficient condition for this is that the functions in the family be uniformly bounded on each compact subset of $G$.

Proposition 1. The unit ball in $E$ is a normal family.

Proof. We must show that the unit ball is uniformly bounded on each compact subset of $G$. Let $K \subset G$ be compact, and let $\lambda_{z}$ denote the functional of evaluation at $z:\left(f, \lambda_{z}\right)=f(z)$ for $f \in E, z \in G$. By Axiom 3 these functionals are bounded. Also, $\sup \left\{\left|\left(f, \lambda_{z}\right)\right|: z \in K\right\}<\infty$ for each $f$. Hence by the principle of uniform boundedness there is a constant $c_{K}$ such that $\left\|\lambda_{z}\right\| \leqslant c_{K}$ for all $z \in K$. Thus $|f(z)| \leqslant c_{K}$ for each $f \in \operatorname{Ball}(E)$, and all $z \in K$.

COROLlARY. If $f_{n} \rightarrow f$ weakly in $E$, then $f_{n}(z) \rightarrow f(z)$ uniformly on each compact subset of $G$. 
We omit the proof. When $E$ is a reflexive Banach space we have a converse to this corollary. First we consider the more general case when $E$ is a conjugate Banach space. We shall take this in the sense of topological isomorphism: there is at least one Banach space $X$ such that $E$ is isomorphic to $X^{*}$. In what follows $X$ will be fixed so that we have a particular weak* topology on $E$.

Proposition 2. Let $E$ be isomorphic to a conjugate Banach space and assume that for each point $z \in G$ the functional of evaluation at $z$ is weak* continuous. Let $\left\{f_{n}\right\} \subset E$ be given. Then the following three statements are equivalent.

(a) $f_{n} \rightarrow 0\left(w_{e a k}^{*}\right)$.

(b)(i) $f_{n}(z) \rightarrow 0$ uniformly on each compact subset of $G$, and (ii) $\left\|f_{n}\right\| \leqslant$ const.

(c)(i) $f_{n}(z) \rightarrow 0(z \in G)$, and (ii) $\left\|f_{n}\right\| \leqslant$ const.

Proof. Proposition 1 shows that (b) and (c) are equivalent. Also, (a) implies (c) trivially. Thus it only remains to show that (b) implies (a). To do this we invoke the theorem that a continuous, one-to-one map of a compact space onto a Hausdorff space is a homeomorphism. For the compact space we take the closed unit ball of $E$, denoted Ball $E$, with the weak* topology. For the Hausdorff space we use Ball $E$ with the topology of uniform convergence on compact subsets of $G$. Our map is the identity. To see that this map is continuous we first note that it is sequentially continuous (since (a) implies (b)). Next note that since $E$ is separable (by Axiom 4), so is any predual of $E$ (see [16, Theorem 2.8.4, p. 34]). Thus Ball $E$ is metrizable in the weak* topology [10, Theorem V.5.1, p. 426] and so it is sufficient to consider sequences. Thus the identity map is a homeomorphism, which completes the proof.

COROLLARY. If $E$ is a reflexive Banach space and if $\left\{f_{n}\right\} \subset E$, then $f_{n} \rightarrow 0$ weakly if and only if both of the following conditions are satisfied: (i) $f_{n}(z) \rightarrow 0(z \in G)$, and (ii) $\left\|f_{n}\right\| \leqslant$ const.

We turn now to a special class of linear transformations on $E$.

Definition. A complex-valued function $\phi$ in $G$ is called a multiplier on $E$ if $\phi E \subset E$.

By $M_{\phi}$ we denote the operator of multiplication by $\phi: M_{\phi} f=\phi f(f \in E)$. The set of all multipliers will be denoted by $M(E)$. An application of the closed graph theorem shows that $M_{\phi}$ is a bounded linear transformation on $E$. Hence it has a finite norm $\left\|M_{\phi}\right\|$. Since $1 \in E$ we have $\phi \in E$ and so $\phi$ is analytic in $G$. The following result shows that multipliers are bounded functions; for a proof see Lemma 11 of [12].

Proposition 3. If $\phi \in M(E)$ then $|\phi(z)| \leqslant\left\|M_{\phi}\right\|, z \in G$.

Notation. $H^{\infty}(G)$ denotes the space of bounded analytic functions in $G$; when $G=\Delta$ we write simply $H^{\infty}$.

Corollary. $M(E) \subset E \cap H^{\infty}(G)$.

Before proceeding we describe the multipliers on the $D_{\alpha}$ spaces. These results are contained in [37 and 38] where, more generally, a complete description of the 
multipliers from $D_{\alpha}$ to $D_{\beta}$ is given, for all $\alpha, \beta$. As usual by the disc algebra $A$ we mean the space of functions continuous on the closed unit disc $\Delta^{-}$and analytic in $\Delta$, with the supremum norm.

1. For $\alpha>1, D_{\alpha}$ is an algebra (see [19, Theorem 3, or 34, Example 1, §9, pp. 99]), and thus $M\left(D_{\alpha}\right)=D_{\alpha}$. Also, $D_{\alpha} \subset A$ (in fact, the power series converge absolutely). It can be shown that the maximal ideal space is the closed unit disc (see [34, Corollary 1 to Proposition 31, p. 94]).

2. For $\alpha \leqslant 0, M\left(D_{\alpha}\right)=H^{\infty}$. (For $\alpha<0$ this follows from (2), and for $\alpha=0$ it follows from the corresponding integral representation of the $H^{2}$ norm.)

3. The case $0<\alpha \leqslant 1$ is considerably more difficult (see [37, Theorem 1.1(c) and 2.3]). The result involves a comparison of the measure $\left|\phi^{\prime}\right|^{2}(1-r)^{1-\alpha} r d r d \theta$ (where $\phi \in M\left(D_{\alpha}\right)$ ) with certain Bessel capacities: when $\alpha=1$ the capacity can be taken to be the ordinary logarithmic capacity. At the end of the present paper there are some further remarks on $M(D)$.

4. For $\alpha \geqslant \beta$ we have $M\left(D_{\alpha}\right) \subset M\left(D_{\beta}\right)$ (see [38, p. 233]).

5. For $\alpha>1$ we have $D_{\alpha} \subset M(D) \subset D \cap H^{\infty}$ (this follows from 1 and 4 above, and from Proposition 3; it can also be proved directly without using 4). In particular, if $\phi^{\prime} \in H^{2}$ (that is, $\phi \in D_{2}$ ) then $\phi \in M(D)$.

6. If $\phi$ is analytic on the closed unit disc $\Delta^{-}$then it is a multiplier on all the $D_{\alpha}$ spaces (this follows from 1 and 4).

7. If $\phi \in H^{\infty}$, then $\phi \in M(D)$ if and only if $\phi^{\prime} D \subset B$. Indeed, $\phi \in M(D)$ if and only if $(\phi f)^{\prime} \in B$, for all $f \in D$. The result now follows since $\phi f^{\prime} \in B$.

We now return to the general theory; as before, let $E$ be a Banach space of analytic functions on a region $G$, that is, Axioms 1-6 are satisfied. A function $f \in E$ is called a cyclic vector (for the operator $M_{z}$ acting on the space $E$ ) if the polynomial multiples of $f$ are dense in $E$. This agrees with the usual terminology in operator theory: a vector $x$ is cyclic for an operator $T$ if the finite linear combinations of the vectors $x, T x, T^{2} x, \ldots$ are dense. Since we shall consider several different spaces of analytic functions we shall sometimes say " $f$ is cyclic for the space $E$ ". Note that the constant function 1 is a cyclic vector for every space $E$, by Axiom 4 .

Proposition 4. If $f$ is cyclic, then $f(z) \neq 0$ for all $z \in G$.

Proof. Let $z_{0} \in G$. The set of all functions in $E$ that vanish at $z_{0}$ is a proper closed subspace of $E$ (Axioms 3 and 4) that is mapped into itself by $M_{z}$.

Question 1. Does there exist a Banach space $E$ of analytic functions for which a function $f$ is cyclic if and only if $f(z) \neq 0$ for all $z \in G$ ?

In such a space the set of cyclic vectors would form a nonempty, relatively closed subset of $E \backslash\{0\}$. The only such example in general operator theory on Banach spaces is Enflo's apparent example of an operator on a (nonreflexive) Banach space with no invariant subspaces (i.e., every nonzero vector is cyclic). This question was first posed in [34] (see Questions 24, 24' in $\$ 11$, and the related discussion of the problem).

It is natural to impose additional conditions on $f$ besides nonvanishing in $G$. In $H^{2}$, for example, Beurling [5] showed that $f$ is cyclic if and only if $f$ has no inner 
factor. The absence of the Blaschke factor is equivalent to the nonvanishing of $f$, and the additional condition is that $f$ have no singular inner factor.

In $D_{\alpha}(\alpha>1), f$ is cyclic if and only if $f$ has no zeros in the closed unit disc, or equivalently,

$$
|f(z)|>c>0 \quad(|z|<1) .
$$

(This follows from the fact that the maximal ideal space is the closed unit disc; one must also show that $f$ is cyclic if and only if it lies in no proper closed ideal. Thus the cyclic vectors are precisely the invertible elements in the Banach algebra $D_{\alpha}$.) For $\alpha=1$ condition (4) is still sufficient for $f$ to be cyclic (see [35]), but is no longer necessary (as we shall see later). Also when $\alpha \leqslant 0$, (4) is sufficient for cyclicity (this follows from Proposition 9).

When $\alpha<0$ some singular inner functions become cyclic. This happens if and only if the associated singular measure puts no mass on any set that is "thin" in the sense of Beurling [4, p. 13], Carleson [8, p. 326] and Hayman [15, Theorems II, IV, pp. 356, 363]. This result was discovered independently by B. Korenblum [22] and J. Roberts (unpublished, but available in an unpublished exposition by Joel Shapiro [33]). Previously, H. S. Shapiro had obtained a partial result [32, Theorem 2]. The thin sets in question are those closed subsets $K$ of $\partial \Delta$ that have Lebesgue measure zero and satisfy $\Sigma\left|I_{n}\right|\left(-\log \left|I_{n}\right|\right)<\infty$. Here $\left\{I_{n}\right\}$ are the disjoint open arcs in the complement of $K$ and $|\cdot|$ denotes normalized Lebesgue measure.

For the Bergman space $(\alpha=-1)$ various sufficient conditions for cyclicity are known, see [34, $\$ 11,12$, especially pp. 11-114 and 120], for more details and references.

We return to the case of a general Banach space $E$ of analytic functions in $G$. If $f \in E$ we let $[f]$ denote the closure in $E$ of the polynomial multiples of $f$. Thus $f$ is cyclic if and only if $[f]=E$.

Proposition 5. Let $f, g \in E$ and let $p$ be a polynomial. Then:

1. $p[f] \subset[f]$.

2. If $g \in[f]$ then $[g] \subset[f]$.

3. If $g \in[f]$ and $g$ is cyclic then $f$ is cyclic.

4. $f$ is cyclic if and only if there exist polynomials $\left\{p_{n}\right\}$ such that $p_{n} f \rightarrow 1$ (in norm).

5. $f$ is cyclic if and only if there exist polynomials $\left\{p_{n}\right\}$ such that $p_{n} f \rightarrow 1$ (weakly).

6. If $E$ is reflexive then $f$ is cyclic if and only if there exist polynomials $\left\{p_{n}\right\}$ such that (i) $p_{n}(z) f(z) \rightarrow 1(z \in G)$, and (ii) $\left\|p_{n} f\right\| \leqslant$ const.

Proof. 1 and 2 are obvious and 2 implies 3 . As to 4 , if $\left\{p_{n}\right\}$ exists with $p_{n} f \rightarrow 1$, then $1 \in[f]$ and so $f$ is cyclic by 3 ; the converse is trivial.

To prove 5 note first that if $f$ is cyclic then by 4 there are polynomials $\left\{p_{n}\right\}$ with $p_{n} f \rightarrow 1$ in norm, and hence weakly. Conversely, if $p_{n} f \rightarrow 1$ weakly then 1 is in the weak closure of the polynomial multiples of $f$. But a subspace is weakly closed if and only if norm closed, so $1 \in[f]$ as required.

Finally, 6 follows from 5 by the Corollary to Proposition 2.

Note that 4, 5 and 6 remain true if 1 is replaced by any other cyclic vector $g$. 
Proposition 6. If $E_{1}, E_{2}$ are two Banach spaces of analytic functions in the same region $G$, and if $E_{1} \subset E_{2}$, then:

(a) $\|f\|_{2} \leqslant c\|f\|_{1}$ for some $c>0$ and all $f \in E_{1}$;

(b) if $f$ is cyclic for $E_{1}$, then it is cyclic for $E_{2}$.

Proof. By Axiom 3 the point evaluation functionals are continuous with respect to both norms. Using this one shows that the identity map of $E_{1}$ into $E_{2}$ has a closed graph and hence is bounded, which proves (a). Part (b) now follows from 4 of Proposition 5.

COROLlaRY 1. If $0 \leqslant \alpha \leqslant 1$, and if $f \in D_{\alpha}$ is cyclic, then $f$ is an outer function.

Proof. Let $E_{1}=D_{\alpha}, E_{2}=H^{2}$.

The converse to this corollary is not valid. Indeed, Carleson has given an example of an outer function in $D_{n}$ (for any positive integer $n \geqslant 2$ given in advance) that is not cyclic in $D$ (see [8, Theorem 6]).

Before stating the next corollary we recall that $H^{1}$ is contained in the Bergman space. This was noted by H. S. Shapiro [32, p. 325], and we recall the proof. If $f \in H^{1}$ then

$$
\|f\|_{B}^{2}=\sum \frac{|\hat{f}(n)|^{2}}{n+1} \leqslant(\max |\hat{f}(k)|) \sum \frac{|\hat{f}(n)|}{n+1}<\pi\left(\|f\|_{H^{1}}\right)^{2}
$$

by the Hardy-Littlewood-Fejér inequality (sometimes called "Hardy's inequality"). See [11, §3.6, p. 48], and for some historical remarks see [36].

COROLlaRY 2. If $f \in H^{1}$ is an outer function, then $f$ is cyclic in $B$.

Several of our results depend on an additional axiom. Recall that $M(E)$ denotes the set of multipliers on $E$.

Axiom 7. $M(E)[f] \subset[f]$, all $f \in E$.

This is equivalent to requiring $M(E) f \subset[f]$.

Question 2. Is Axiom 7 a consequence of Axioms 1-6?

This seems unlikely though at present we have no counterexample. See the end of the paper for further comments and a possible example.

Proposition 7. Each of the spaces $D_{\alpha}(-\infty<\alpha<\infty)$ satisfies Axiom 7.

Proof. Fix $\alpha$ and let $\phi \in M\left(D_{\alpha}\right)$. By Theorem 12(iii) of [34] there is a sequence of polynomials (the Fejér means of the partial sums of the power series for $\phi$ ) such that $p_{n} f \rightarrow \phi f$ in norm, for each $f \in D_{\alpha}$ (i.e., $\left\{p_{n}\right\}$ converges to $f$ in the strong operator topology). Hence $\phi f \in[f]$, as required.

From now on we shall assume that our space $E$ satisfies Axiom 7.

Proposition 8. If $f \in E$ and $\phi \in M(E)$ then $\phi f$ is cyclic and if and only if both $f$ and $\phi$ are cyclic.

Proof. First assume that both $f$ and $\phi$ are cyclic, and choose polynomials $\left\{p_{n}\right\}$ such that $p_{n} f \rightarrow 1$. Then $p_{n}(\phi f) \rightarrow \phi$, so $\phi \in[\phi f]$; since $\phi$ is cyclic this proves that $\phi f$ is cyclic. 
Conversely, assume that $\phi f$ is cyclic, and choose polynomials $\left\{p_{n}\right\}$ such that $p_{n} \phi f \rightarrow 1$. By Axiom 7 we have $p_{n} \phi f \in[f]$, hence $1 \in[f]$, and so $f$ is cyclic. Now choose polynomials $\left\{q_{n}\right\}$ such that $q_{n} \rightarrow f$ in $E$. Then $q_{n} \phi \rightarrow \phi f$ and so the cyclic vector $\phi f$ is in $[\phi]$, and thus $\phi$ is cyclic.

Note that Axiom 7 was only used to show that if $\phi f$ is cyclic then so is $f$. For the next result we recall that $H^{1} \subset B$.

COROLLARY. If $f \in H^{1}$ then $f$ is cyclic for the Bergman space if and only if its inner factor is cyclic, that is, if and only if there is no Blaschke factor and the singular factor satisfies the Korenblum-Roberts condition.

Proof. The proposition is applicable since $B$ admits all bounded functions as multipliers. The result now follows from what has gone before.

We now pose two questions about cyclic vectors in a general Banach space $E$ of analytic functions in a region $G$.

Question 3. If $f, g \in E$, if $g$ is cyclic, and if $|f(z)| \geqslant|g(z)|$ for all $z \in G$, then must $f$ be cyclic?

Since the constant function 1 is always a cyclic vector, we have the following special case of this question.

Question $3^{\prime}$. If $f \in E$ and $|f(z)|>c>0$ for all $z \in G$, must $f$ be cyclic?

Question 4. If $f, f^{-1} \in E$, must $f$ be cyclic?

This question (for the Bergman space) was posed in [34] (see Question 25' on p. 114). Harold S. Shapiro [31] used the term "weakly invertible" in place of cyclic. The above question could then be rephrased as follows: does invertibility imply weak invertibility? From Proposition 8 we see that the answer is affirmative if, in addition, we assume that $f$ is a multiplier. Indeed, $1=f f^{-1}$ is always cyclic.

Thus far no examples are known where either of these two questions has a negative answer. There is one common situation where Question 3 has an affirmative answer.

Proposition 9. If $M(E)=H^{\infty}(G)$, if $f, g \in E$ with $|f(z)| \geqslant|g(z)|$ for all $z \in G$, and if $g$ is cyclic, then $f$ is cyclic.

Proof. Since $\phi=g / f$ is bounded it is a multiplier on $E$. Hence $g=\phi f \in[f]$ by Axiom 7, and so $f$ is cyclic.

We now consider Questions 3 and 4 for the spaces $D_{\alpha}$. When $\alpha>1$, then, as remarked earlier, $f$ is cyclic if and only if it has no zeros in the closed unit disc (recall that the functions are continuous on the closed disc). It follows easily that both questions can be answered in the affirmative.

Both questions are open when $0<\alpha \leqslant 1$; later we give a partial answer to Question 3 when $\alpha=1$ (see Theorem 1 and Corollary 1, as well as Corollary 2 to Theorem 2).

For $\alpha \leqslant 0$ Proposition 9 gives an affirmative answer to Question 3. For $\alpha=0$ Question 4 can also be answered in the affirmative: if $f$ and $f^{-1}$ are both in $H^{2}$ then they are both outer functions (indeed, if either $f$ or $f^{-1}$ had a nontrivial inner factor, then so would $f^{-1} f=1$ ). Question 4 is open for $\alpha<0$. This leads to another 
interesting problem, where for simplicity we specialize to the Bergman space $B$ $(\alpha=-1)$. If $f \in B$ then for $r=|z|<1$

$$
|f(z)|^{2}=\left|\sum \hat{f}(n)(n+1)^{-1 / 2}(n+1)^{1 / 2} z^{n}\right|^{2} \leqslant\|f\|^{2}\left(1-r^{2}\right)^{-2} .
$$

Hence if $f^{-1} \in B$ then $|f(z)| \geqslant\left\|f^{-1}\right\|^{-1}\left(1-r^{2}\right)$. This suggests the following question, which was first posed by H. S. Shapiro (see the Remark following Theorem 5 in [32]).

Question 5. If $f \in B$ and if $|f(z)|>c(1-|z|)^{k}$ for some $c, k>0$ (and all $z \in \Delta$ ), then must $f$ be cyclic?

As noted above, an affirmative answer to this question would imply an affirmative answer to Question 4 for the Bergman space. Partial results are given in $[31,32,1]$ and also in [34] (see $\$ \S 11,12$, Propositions 41, 43 and Questions 25, 25').

Along these lines one can pose similar questions for any Banach space of analytic functions on a bounded plane domain $G$. We consider continuous functions $\phi$ on $G^{-}$ (the closure of $G$ ) such that $\phi$ is positive on $G$ and zero on $\partial G$.

Question 6. Let $E$ be a Banach space of analytic functions on $G$. Does there exist a function $\phi$, as above, such that if $f \in E$ satisfies $|f(z)|>\phi(z)$ for all $z \in G$, then $f$ is cyclic?

Such functions exist for the $D_{\alpha}$ spaces when $\alpha>1$. Indeed, by Proposition 10(b), if $f \in D_{\alpha}(1<\alpha<3)$ then $f$ satisfies a Lipschitz condition of order $b=(\alpha-1) / 2$. Hence if $f$ has a zero on the boundary, say $f(1)=0$, then

$$
|f(z)|=|f(z)-f(1)| \leqslant c|z-1|^{b}, \quad|z| \leqslant 1 .
$$

Hence if $\phi(z)=(1-|z|)^{\alpha}$ where $0<a<b$, and if $f \in D_{\alpha}$ satisfies $|f(z)| \geqslant \phi(z)$ in $\Delta$, then $f$ has no zeros on the closed unit disk and thus is cyclic. Of course, if $\phi$ works for $D_{\alpha}$ then it also works for $D_{\beta}$ for all $\beta>\alpha$, since $D_{\beta} \subset D_{\alpha}$.

In contrast to this, such functions $\phi$ do not exist for the space $H^{2}$. Indeed, H. S. Shapiro has shown (see [31, p. 164]) that if $S(z)$ is a singular inner function with associated singular measure $\mu$, then

$$
|S(z)| \geqslant \exp \left\{-c \frac{\omega(1-r)}{1-r}\right\} \quad(r=|z|<1),
$$

where $\omega$ is the modulus of continuity of $\mu$, and $c$ is a positive constant. Also, one can construct singular measures $\mu$ for which $\omega(\delta) / \delta$ tends to infinity arbitrarily slowly (see [43, $\S 5$ and 44, p. 265]). Thus, given any function $\phi$ as above, there exists a singular inner function $S$ such that $|S(z)| \geqslant \phi(z)$ for $|z|<1$.

Question 6 is open for the spaces $D_{\alpha}$ in the remaining intervals $0<\alpha \leqslant 1$ and $\alpha<0$.

We consider the function $(1-z)^{-\alpha}=\sum a_{n}(\alpha) z^{n}$. Here $\alpha$ is complex, $|z|<1$, and we use the branch with $a_{0}(\alpha)=1$. By a formula of Euler for the gamma function (see the example in $\$ 12.11$, p. 237 of [40]) we have

$$
a_{n}(\alpha)=\frac{\alpha(\alpha+1) \cdots(\alpha+n-1)}{n !} \sim \frac{n^{\alpha-1}}{\Gamma(\alpha)} .
$$


Proposition 10. (a) If $g \in D_{\beta}(\beta<1)$ then

$$
|g(z)| \leqslant c\|g\|_{\beta}(1-|z|)^{-(1-\beta) / 2} \quad(|z|<1),
$$

where $c$ depends only on $\beta$, not on $g$.

(b) If $f \in D_{\alpha}(1<\alpha<3)$, then

$$
|f(z)-f(w)| \leqslant c\|f\|_{\alpha}|z-w|^{(\alpha-1) / 2} \quad(|z| \leqslant 1,|w| \leqslant 1),
$$

where $c$ depends only on $\alpha$, not on $f$.

Proof. (a) We have (with $r=|z|$ )

$$
|g(z)|^{2}=\left|\sum \hat{g}(n)(n+1)^{\beta / 2} z^{n}(n+1)^{-\beta / 2}\right|^{2} \leqslant\|g\|_{\beta}^{2} \sum(n+1)^{-\beta} r^{2 n} .
$$

The result now follows from (5) above.

(b) We have $f^{\prime} \in D_{\alpha-2}$ and $\left\|f^{\prime}\right\|_{\alpha-2} \leqslant\|f\|_{\alpha}$.

A theorem of Hardy and Littlewood (see [11, Theorem 5.1, p. 74]), says that $f$ (analytic in $\Delta$ ) satisfies a Lipschitz condition of order $b(0<b<1)$ in $\Delta$ if and only if $\left|f^{\prime}(z)\right| \leqslant c(1-|z|)^{b-1}$. The result now follows from (a).

2. The Dirichlet space. In this section we shall give an affirmative answer to Question 3, under various additional hypotheses. We require some lemmas; the first two are proved in [35].

LEMMA 1. If $f \in D, r=|z|$ and $0 \leqslant t \leqslant 1$, then

(a) $|f(z)-f(t z)|^{2} \leqslant\|f\|^{2} \log ((1-r t) /(1-r))(r<1)$,

(b) $|f(z)-f(t z)|^{2} \leqslant\|f\|^{2} \log 2(r \leqslant t)$.

LEMMA 2. If $0<t<1$ then

$$
\int_{t}^{1} \frac{1}{1-r t} \log \frac{1-r t}{1-r} d r \leqslant 2 \log 2 .
$$

Notation. $h_{t}(z)=h(t z), 0 \leqslant t \leqslant 1$.

The integrals below are with respect to area measure on $\Delta$. Let $\|h\|_{B}^{2}=\pi^{-1} \iint|h|^{2}$; this is the Bergman norm of $h$ (see (3)).

LEMMA 3. If $f, g \in D$ then:

(a) $\pi^{-1} \iint\left|f-f_{t}\right|^{2}\left|\left(g_{t}\right)^{\prime}\right|^{2} \leqslant(5 \log 2)\|f\|^{2}\left\|g^{\prime}\right\|_{B}^{2}, 0<t<1$,

(b) $\lim _{t \uparrow 1} \iint\left|f-f_{t}\right|^{2}\left|\left(g_{t}\right)^{\prime}\right|^{2}=0$.

Proof. (a) Since $\left(g_{t}\right)^{\prime}(z)=\operatorname{tg}^{\prime}(t z)$ we have $\left|\left(g_{t}\right)^{\prime}(z)\right| \leqslant\left|g^{\prime}(t z)\right|$. Let

$$
a(r)=\int_{0}^{2 \pi}\left|g^{\prime}\left(r e^{i \theta}\right)\right|^{2} d \theta .
$$

This is an increasing function of $r$ (this is obvious if one expresses the integral in terms of the Taylor coefficients of $g$ ). Hence $\iint\left|\left(g_{t}\right)^{\prime}\right|^{2} \leqslant \iint\left|g^{\prime}\right|^{2}, 0<t<1$. The integral on the right is finite since $g^{\prime}$ is in the Bergman space. Thus by Lemma 1(b),

$$
\pi^{-1} \int_{0}^{2 \pi} \int_{0}^{t}\left|f-f_{t}\right|^{2}\left|\left(g_{t}\right)^{\prime}\right|^{2} \leqslant(\log 2)\|f\|^{2}\left\|g^{\prime}\right\|_{B}^{3} \text {. }
$$


Since $a(r)$ is increasing we have

$$
\frac{1}{2}(1-r) a(r) \leqslant \int_{r}^{1} a(s) s d s \leqslant \pi\left\|g^{\prime}\right\|_{B}^{2} .
$$

Thus by Lemma 1(a),

$$
\begin{aligned}
\int_{0}^{2 \pi} \int_{t}^{1}\left|f-f_{t}\right|^{2}\left|\left(g_{t}\right)^{\prime}\right|^{2} & \leqslant\|f\|^{2} \int_{t}^{1} a(t r) \log \frac{1-r t}{1-r} d r \\
& \leqslant 2 \pi\|f\|^{2}\left\|g^{\prime}\right\|_{B}^{2} \int_{t}^{1} \frac{1}{1-r t} \log \frac{1-r t}{1-r} d r
\end{aligned}
$$

Inequality (a) now follows from Lemma 2.

(b) First note that (b) is valid when $f$ is a polynomial (and $g$ is arbitrary). Now let $f, g$ both be arbitrary and let $\varepsilon>0$ be given. Choose a polynomial $p$ such that $\|f-p\|<\varepsilon$ and let $h=f-p$. Then

$$
\iint\left|f-f_{t}\right|^{2}\left|\left(g_{t}\right)^{\prime}\right|^{2} \leqslant \iint\left|p-p_{t}\right|^{2}\left|\left(g_{t}\right)^{\prime}\right|^{2}+\iint\left|h-h_{t}\right|^{2}\left|\left(g_{t}\right)^{\prime}\right|^{2} .
$$

Therefore from part (a) we have

$$
\varlimsup_{t \uparrow 1} \iint\left|f-f_{t}\right|^{2}\left|\left(g_{t}\right)^{\prime}\right|^{2} \leqslant(5 \log 2) \varepsilon^{2} \pi\left\|g^{\prime}\right\|_{B}^{2} .
$$

The result follows since $\varepsilon$ was arbitrary.

In the next lemma we shall use the obvious fact that if $f$ is bounded and $h$ is in the Bergman space, then $\|f h\|_{B} \leqslant\|f\|_{\infty}\|h\|_{B}$.

LEMMA 4. The space $H^{\infty} \cap D$ is a Banach algebra with the norm $\|f\|_{*}=\|f\|_{\infty}+$ $\left\|f^{\prime}\right\|_{B}$.

Proof. Let $f, g \in H^{\infty} \cap D$. Then

$$
\|f g\|_{*}=\|f g\|_{\infty}+\left\|f g^{\prime}+f^{\prime} g\right\|_{B} \leqslant\|f\|_{\infty}\|g\|_{\infty}+\|f\|_{\infty}\left\|g^{\prime}\right\|_{B}+\left\|f^{\prime}\right\|_{B}\|g\|_{\infty} \leqslant\|f\|_{*}\|g\|_{*} .
$$

Also, the identity element has norm one: $\|1\|=1$.

In particular, this lemma tells us that if $g \in H^{\infty} \cap D$ then $g^{2} \in D$. We now give a partial answer to Question 3. We require the following fact:

$$
\|f\|_{D}^{2} \leqslant 2\left\|f^{\prime}\right\|_{B}^{2}+|f(0)|^{2} \quad(f \in D) .
$$

This may be seen by expressing the norm in terms of Taylor coefficients.

THEOREM 1. If $f \in D, g \in H^{\infty} \cap D,|f(z)| \geqslant|g(z)|$ in $\Delta$, and if $g^{2}$ is cyclic, then $f$ is cyclic.

Proof. It will be sufficient to show that $g^{2} \in[f]$ (recall that $[f]$ denotes the closure of the polynomial multiples of $f$ ). Since $g_{t}^{2} / f_{t}$ is analytic on the closed unit disc (for $t<1$ ), it is a multiplier on all of our spaces, in particular on $D$. Hence by Axiom 7 (and Proposition 7), $\left(g_{t}^{2} / f_{t}\right) f \in[f]$. We shall show that these functions converge in norm to $g^{2}$ as $t \rightarrow 1$. Since $\left(g_{t}^{2} / f_{t}\right) f-g^{2}$ vanishes at the origin, it will 
be sufficient, in view of (6), to consider the Bergman norm of the derivative. Also since $g_{t}^{2}$ converges in norm to $g^{2}$, we may replace $g$ by $g_{t}$. Then we have

$$
\frac{d}{d z}\left\{\left(\frac{f}{f_{t}}-1\right) g_{t}^{2}\right\}=\left(\frac{f-f_{t}}{f_{t}}\right)^{\prime} g_{t}^{2}+2 \frac{f-f_{t}}{f_{t}} g_{t}\left(g_{t}\right)^{\prime}=\phi_{1}+\phi_{2} .
$$

First, $\left|\phi_{2} / 2\right| \leqslant\left|f-f_{t}\right|\left|\left(g_{t}\right)^{\prime}\right| ;$ hence $\left\|\phi_{2}\right\|_{B} \rightarrow 0$ as $t \rightarrow 1$, by Lemma 3(b).

Next,

$$
\phi_{1}=\frac{f_{t}\left(f-f_{t}\right)^{\prime}}{f_{t}^{2}} g_{t}^{2}-\frac{\left(f-f_{t}\right)\left(f_{t}\right)^{\prime}}{f_{t}^{2}} g_{t}^{2}=\phi_{3}+\phi_{4} .
$$

Then $\left|\phi_{4}\right| \leqslant\left|\left(f-f_{t}\right)\left(f_{t}\right)^{\prime}\right|$ and so $\left\|\phi_{4}\right\|_{B} \rightarrow 0$ by Lemma 3(b).

Finally, $\left|\phi_{3}\right| \leqslant\left|\left(f-f_{t}\right)^{\prime} g_{t}\right|$. Hence

$$
\left\|\phi_{3}\right\|_{B} \leqslant\left\|\left(f-f_{t}\right)^{\prime}\right\|_{B}\left\|g_{t}\right\|_{\infty},
$$

and so $\|\phi\|_{B} \rightarrow 0$, which completes the proof.

Note that the boundedness of $g$ was used only at the end of the proof (in estimating $\left.\phi_{3}\right)$. It would not be needed at all if one could show that $\left\|\left(f-f_{t}\right)^{\prime} g_{t}\right\|_{B} \rightarrow 0$ as $t \rightarrow 1$, whenever $f, g \in D$. However this is not true in general, as the following example shows. In this example we have $g^{2} \in D, g^{2}$ is cyclic (as are $f$ and $g$ ), $|f(z)| \geqslant c|g(z)|$ for $z \in \Delta$, and the above norm tends to infinity as $t \rightarrow 1$.

EXAMPLE 1. Let $h(z)=-z^{-1} \log (1-z)$, let $f=h^{\alpha}$ and $g=h^{\beta}$; here $0<\beta<\alpha$ are to be determined. It can be shown that $h^{a} \in D$ (equivalently, $\left(h^{a}\right)^{\prime} \in B$ ) if and only if $a<\frac{1}{2}$. Thus $f, g, g^{2} \in D$ if $\alpha<\frac{1}{2}$ and $\beta<\frac{1}{4}$. Since $|h(z)|>c>0$ in $\Delta$ we see (as noted earlier) that $f, g, g^{2}$ are all cyclic, and that $|f| \geqslant c|g|$ in $\Delta$.

Note. Here, and in what follows, $c$ will denote a general constant, not necessarily the same at each occurrence.

Let $S_{t}$ denote the region exterior to the disc $|z| \leqslant t$ but inside the triangle with vertices at $1,(1 \pm i) / 2$. We shall only be interested in this for $t$ near 1 . Then

$$
\left\|\left(f-f_{t}\right)^{\prime} g_{t}\right\|_{B} \geqslant\left\{\iint_{S_{t}}\left|\left(f-f_{t}\right)^{\prime} g_{t}\right|^{2}\right\}^{1 / 2} \geqslant\left\{\iint_{S_{t}}\left|f^{\prime} g_{t}\right|^{2}\right\}^{1 / 2}-\left\{\iint_{S_{t}}\left|f_{t}^{\prime} g_{t}\right|^{2}\right\}^{1 / 2}
$$

We shall show that the first term on the right side tends to infinity while the second term tends to zero, as $t \rightarrow 1$.

It can be shown that for $z \in S_{t}$ (and $t$ near 1) we have

$$
\left|f_{t}^{\prime}(z) g_{t}(z)\right| \leqslant c(1-t)^{-1}[-\log (1-t)]^{\alpha+\beta-1} .
$$

Since area $\left(S_{t}\right) \leqslant c(1-t)^{2}$, we see that the second term on the right side of (7) tends to zero, as asserted.

Also, it can be shown that for $z \in S_{t}$ and $r=|z|$ we have

$$
\left|f^{\prime}(z) g_{t}(z)\right| \geqslant c(1-r)^{-1}[-\log (1-r)]^{\alpha-1}[-\log (1-t)]^{\beta} .
$$


We express the area integral in polar coordinates and integrate first with respect to $d \theta$. This contributes at least $c(1-r)$. Thus we have

$$
\begin{aligned}
\iint_{S_{t}}\left|f^{\prime} g_{t}\right|^{2} & \geqslant c[-\log (1-t)]^{2 \beta} \int_{t}^{1}(1-r)^{-1}[\log (1-r)]^{2 \alpha-2} d r \\
& \geqslant c[-\log (1-t)]^{2 \beta+2 \alpha-1} .
\end{aligned}
$$

This tends to infinity if $\alpha+\beta>\frac{1}{2}$. To complete our example we may choose $\alpha=2 / 5, \beta=1 / 5$.

Note that in this example $f^{\prime} g \notin B$. Perhaps in case $f^{\prime} g \in B$ the norm in (7) does tend to zero; if so this would mean that the conclusion of Theorem 1 would be valid when we replace the assumption that $g \in H^{\infty} \cap D$ by the (weaker) assumptions: $g$, $g^{2} \in D, g f^{\prime} \in B$. (The remaining hypotheses of the theorem are carried over unchanged.)

We now give two corollaries to Theorem 1 .

CoRollary 1. If $f \in D, g \in M(D),|f(z)| \geqslant|g(z)|$ in $\Delta$, and if $g$ is cyclic, then $f$ is cyclic.

Proof. It follows from Propositions 3 and 8 that $g \in H^{\infty}$ and that $g^{2}$ is cyclic.

Corollary 2. If $g \in H^{\infty} \cap D$ and if $g^{2}$ is cyclic, then $g$ is cyclic.

Proof. Take $f=g$ in Theorem 1. Note that this corollary also follows from Proposition 11.

One might ask whether Corollary 1 is not equivalent to the theorem. That is, if $g \in H^{\infty} \cap D$ is cyclic, then must $g$ be a multiplier? The following example shows that this is not the case.

EXAmple 2. There exists a function $g \in D$ that is cyclic and has an absolutely convergent power series but is not a multiplier. Indeed, in [38] G. D. Taylor gave an example of a function $f$ in $D$, having an absolutely convergent power series that is not a multiplier. (In [37] D. Stegenga gave an example of a function in $D$ that is also in the disc algebra but is not a multiplier.) If $c$ is a large constant, then the function $g=f+c$ will, in addition, be bounded away from 0 in $\Delta$ and hence will be cyclic. (Taylor's example has the further property that $\hat{f}(n) \downarrow 0$.)

It seems unnatural in Theorem 1 to assume that $g$ is bounded; indeed, the hypothesis says that $f$ does not approach zero too rapidly, and this will be true all the more if $g$ is unbounded. Also, it seems unpleasant to assume that $g^{2}$ (rather than $g$ ) is cyclic. These remarks suggest the following questions.

Question 7. If $f, g \in D$ with $g$ cyclic, and if $|f(z)| \geqslant|g(z)|$ in $\Delta$, then does there exist $g_{1} \in H^{\infty} \cap D$, with $g_{1}$ cyclic and $|f(z)| \geqslant\left|g_{1}(z)\right|$ in $\Delta$ ?

Question 8. If $g \in H^{\infty} \cap D$ is cyclic, must $g^{2}$ be cyclic? More generally, if $f$, $g \in H^{\infty} \cap D$ are both cyclic, must $f g$ be cyclic?

We indicate a method to attack this problem, but first we establish the converse.

Proposition 11. If $f, g \in H^{\infty} \cap D$, and if $f g$ is cyclic, then both $f$ and $g$ are cyclic. 
Proor. Since $f$ and $g$ occur symmetrically it will be sufficient to show that $g$ is cyclic; we do this by showing that $f g \in[g]$. Let $\left\{\sigma_{n}\right\}$ denote the Fejér means of the partial sums of the power series for $f$. Since the partial sums converge to $f$ in $D$ the same will be true for $\left\{\sigma_{n}\right\}$. We claim that $\sigma_{n} g \rightarrow f g$ in $D$.

Indeed, first note that $\sigma_{n} g \rightarrow f g$ pointwise in $\Delta$. Then by (6), it will be sufficient to prove that $\left.\| \sigma_{n} g-f g\right)^{\prime} \|_{B} \rightarrow 0$. We have

$$
\left\|\left(\sigma_{n} g-f g\right)^{\prime}\right\|_{B} \leqslant\left\|\left(\sigma_{n}-f\right) g^{\prime}\right\|_{B}+\left\|\left(\sigma_{n}-f\right)^{\prime}\right\|_{B}\|g\|_{\infty} .
$$

The first term on the right tends to zero by the dominated convergence theorem since $\left\|\sigma_{n}-f\right\|_{\infty} \leqslant 2\|f\|_{\infty}$. As to the second term,

$$
\left\|\left(\sigma_{n}-f\right)^{\prime}\right\|_{B} \leqslant\left\|\sigma_{n}-f\right\|_{D} \rightarrow 0,
$$

which completes the proof.

This proposition allows us to answer Question 4 (for the space $D$ ) in the speical case when both $f$ and $1 / f$ are bounded.

COROLlaRY. If $f, 1 / f \in H^{\infty} \cap D$, then both are cyclic.

Is this conclusion valid if we drop the hypothesis that $f$ be bounded $(1 / f$ is still required to be bounded)? We restate this as follows.

Question 9. If $f \in D$ and if $|f(z)| \geqslant c>0$ in $\Delta$, then must $1 / f$ be cyclic?

These hypotheses imply that $1 / f \in H^{\infty} \cap D$ (the fact that $(1 / f)^{\prime} \in B$ follows from the inequality $\left.\left|(1 / f)^{\prime}\right| \leqslant\left|f^{\prime}\right| / c^{2}\right)$. Also, $f$ must be cyclic (this is the main result of [35], and it is also an immediate consequence of Theorem 1).

Lemma 5.If $f, g \in H^{\infty} \cap D$, if $f$ is cyclic, and if there exist polynomials $\left\{p_{n}\right\}$ such that:

(a) $p_{n}(z) g(z) \rightarrow 1(z \in \Delta)$,

(b) $\left\|p_{n} g\right\|_{\infty} \leqslant$ const,

(c) $\left\|p_{n} g\right\|_{D} \leqslant$ const, then $f g$ is cyclic.

Proof. It is sufficient to show that $f \in[f g]$. Since $p_{n} f g \rightarrow f$ pointwise in $\Delta$, it suffices to show that $\left\|p_{n} f g\right\|_{D} \leqslant$ const (see Proposition 5 and the remark following the proof). By (6) it is sufficient to show that $\left\|\left(p_{n} f g\right)^{\prime}\right\|_{B} \leqslant$ const. We have

$$
\left\|\left(\left(p_{n} g\right) f\right)^{\prime}\right\|_{B} \leqslant\left\|p_{n} g\right\|_{\infty}\left\|f^{\prime}\right\|_{B}+\|f\|_{\infty}\left\|\left(p_{n} g\right)^{\prime}\right\|_{B},
$$

which is bounded since $\left\|\left(p_{n} g\right)^{\prime}\right\|_{B} \leqslant\left\|p_{n} g\right\|_{D}$. This completes the proof.

We do not know whether such polynomials always exist (that is, for every cyclic $g$ in $H^{\infty} \cap D$ ). Perhaps this would be true if $g \in A \cap D$ (where $A$ is the disc algebra). However, we are only able to prove this under the stronger hypothesis that $|g|$ is Dini continuous on $\partial \Delta$.

In what follows we shall modify an unbounded function $f$ by multiplying it by the "cut-off" function $\phi_{f}$ defined below. We assume that $f \in H^{2}$. We define $\phi_{f}$ to be the outer function

$$
\phi(z)=\phi_{f}(z)=\exp \left\{\frac{1}{2 \pi} \int_{0}^{2 \pi} \frac{e^{i t}+z}{e^{i t}-z} \log \left|\phi_{f}\left(e^{i t}\right)\right| d t\right\}, \quad|z|<1,
$$


where $\left|\phi_{f}\left(e^{i t}\right)\right|=1$ whenever $\left|f\left(e^{i t}\right)\right| \leqslant 1$, and $\left|\phi_{f}\right|=|f|^{-1}$ otherwise. Then $\phi \in H^{\infty}$ and $\|\phi\|_{\infty} \leqslant 1$. If we have a sequence of functions $\left\{f_{n}\right\}$, then we shall write $\left\{\phi_{n}\right\}$ for the corresponding sequence of functions defined by (8).

LEMMA 6. If $\left\{f_{n}\right\} \subset H^{2}$, and if $f_{n} \rightarrow 1$ in norm, then $\phi_{n}(z) \rightarrow 1$ in $\Delta$, uniformly on compact sets.

Proof. Since $\left\|\phi_{n}\right\|_{\infty} \leqslant 1$ for all $n$, it is sufficient to show that $\phi_{n}(0) \rightarrow 1$. This is equivalent to showing that

$$
\lim _{n \rightarrow \infty} \int_{0}^{2 \pi} \log \left|\phi_{n}\left(e^{i t}\right)\right| d t=0,
$$

that is, $\left\{\log \left|\phi_{n}\right|\right\}$ converges to 0 in $L^{1}(\partial \Delta)$. Let $\varepsilon>0$ be given and let

$$
\begin{aligned}
& E_{n}=\left\{w \in \partial \Delta:\left|f_{n}(w)\right| \geqslant 1+\varepsilon\right\}, \\
& F_{n}=\left\{w \in \partial \Delta: 1<\left|f_{n}(w)\right|<1+\varepsilon\right\} .
\end{aligned}
$$

Then $\left|\phi_{n}\right|=\left|f_{n}\right|^{-1}$ on $E_{n} \cup F_{n}$, and $\left|\phi_{n}\right|=1$ otherwise. Then

$$
\begin{gathered}
\int-\log \left|\phi_{n}\right|=\int_{E_{n}} \log \left|f_{n}\right|+\int_{F_{n}} \log \left|f_{n}\right|=\mathrm{I}_{n}+\mathrm{II}_{n}, \\
\mathrm{II}_{n} \leqslant\left|F_{n}\right| \log (1+\varepsilon) \leqslant 2 \pi \varepsilon, \quad \mathrm{I}_{n} \leqslant \int_{E_{n}}\left|f_{n}\right| \leqslant\left|E_{n}\right|^{1 / 2}\left\|f_{n}\right\|_{H^{2}} .
\end{gathered}
$$

Since $f_{n} \rightarrow 1$ in $L^{2}$ we have $f_{n} \rightarrow 1$ in measure. Since

$$
\left\{\left|f_{n}\right| \geqslant 1+\varepsilon\right\} \subset\left\{\left|f_{n}-1\right| \geqslant \varepsilon\right\}
$$

we see that $\left|E_{n}\right| \rightarrow 0$ as $n \rightarrow \infty$ (for each fixed $\varepsilon$ ). The result follows.

Before proceeding we recall a formula of Carleson [9] for the Dirichlet integral of a function $f$ (that is, for $\left\|f^{\prime}\right\|_{B}^{2}$ ). This formula is the sum of three nonnegative terms, involving respectively the Blaschke factor of $f$, the singular inner factor, and the outer factor. We reproduce only the third of these, as inequality (10) below. We shall write $f(t)$ instead of $f\left(e^{i t}\right)$ for the boundary values of $f$. To simplify subsequent formulae we introduce the following notation:

(9) $I(f)=I(f ; x, t)=(\log |f(x+t)|-\log |f(x)|)\left(|f(x+t)|^{2}-|f(x)|^{2}\right)$.

Then from Carleson's formula we have

$$
(8 \pi)^{-1} \int_{0}^{\pi}\left(\sin \frac{1}{2} t\right)^{-2} d t \int_{-\pi}^{\pi} I(f ; x, t) d x \leqslant\left\|f^{\prime}\right\|_{B}^{2} \quad(f \in D),
$$

with equality when $f$ is an outer function. Note that $I(f ; x, t)$ is nonnegative for all $x, t$ since the two terms on the right side of (9) have the same sign. Hence $I(f)$ is unchanged if we replace each of these terms by its absolute value.

A continuous function $g$ on $\partial \Delta$ has a modulus of continuity

$$
\omega(\delta)=\omega_{g}(\delta)=\sup \{|f(z)-f(w)|:|z-w| \leqslant \delta, z, w \in \partial \Delta\} .
$$

If $g \in A$ (the disc algebra), then there is a corresponding modulus of continuity where $z, w \in \Delta^{-}$. This modulus can be strictly larger than the boundary modulus 
defined above, but is less than 3 times the boundary modulus (see [28]). A continuous function $g$ is said to be Dini continuous if

$$
\int_{0}^{\pi} \frac{\omega(\delta)}{\delta} d \delta<\infty
$$

In particular this is true when $g$ satisfies a Lipschitz condition (i.e., $\omega(\delta) \leqslant c \delta^{\alpha}$ for some $\alpha>0$ ). We only need the case where $g=|f|$ and $f \in A$. Trivially one has $\omega_{|f|} \leqslant \omega_{f}$; thus $|f|$ is Dini continuous whenever $f$ is.

Lemma 7. Let $\left\{f_{n}\right\} \subset D$ with $f_{n} \rightarrow 1$ in norm, and let $\left\{\phi_{n}\right\}$ be the associated functions defined by (8). Then:

(a) $\left\{\phi_{n}\right\} \subset D \cap H^{\infty}$.

(b) $\left(\phi_{n} f_{n}\right)(z) \rightarrow 1$ for $z \in \Delta$ (uniformly on compact subsets).

(c) $\left\|\phi_{n} f_{n}\right\|_{\infty} \leqslant 1$ for all $n$.

(d) $\left\|\phi_{n} f_{n}\right\|_{D} \leqslant\left\|f_{n}\right\|_{D} \leqslant$ const for all $n$.

(e) If $\left|f_{n}\right|$ is Dini continuous on $\partial \Delta$ then $\phi_{n}$ is in the disc algebra.

Proof. We prove the assertions in this order: (b), (c), (a), (d), (e).

(b) We have $f_{n}(z) \rightarrow 1$ and $\phi_{n}(z) \rightarrow 1$ uniformly on compact sets by the Corollary to Proposition 1 and by Lemma 6, and the result follows.

For the remainder of the proofs we shall omit the subscript $n$.

(c) Since $\phi \in H^{\infty}$ and $f \in D \subset H^{2}$ we see that $\phi f \in H^{2}$. In addition, $|\phi f| \leqslant 1$ almost everywhere on $\partial \Delta$, and so $\phi f$ is in the unit ball of $H^{\infty}$, as was to be proved.

(a) We already know that $\|\phi\|_{\infty} \leqslant 1$ so we only need to show that $\phi \in D$. We shall show that $\left\|\phi^{\prime}\right\|_{B} \leqslant\left\|f^{\prime}\right\|_{B}$ which, by (6), will complete the proof. Since $\phi$ is an outer function we may compute $\left\|\phi^{\prime}\right\|_{B}$ from (10). Thus it will be sufficient to prove that $I(\phi) \leqslant I(f)$ for all $x, t$.

First we show that

$$
\left.|| \phi(x+t)\right|^{2}-|\phi(x)|^{2}|\leqslant||f(x+t)|^{2}-|f(x)|^{2} \mid .
$$

We consider the following four cases.

(i) $|f(x+t)| \leqslant 1$ and $\mid f(x) \leqslant 1$. In this case $|\phi(x+t)|=|\phi(x)|=1$ and (12) follows.

(ii) $|f(x+t)|>1$ and $|f(x)|>1$. Then $|\phi|=|f|^{-1}$ and so

$$
\left.|| \phi(x+t)\right|^{2}-|\phi(x)|^{2}|=| \frac{|f(x)|^{2}-|f(x+t)|^{2}}{|f(x+t)|^{2}|f(x)|^{2}}|\leqslant||f(x)|^{2}-|f(x+t)|^{2} \mid .
$$

(iii) $|f(x+t)| \leqslant 1$ and $|f(x)|>1$. Then

$$
\left.|| \phi(x+t)\right|^{2}-\left.|\phi(x)|^{2}|=1-| f(x)\right|^{-2} \leqslant|f(x)|^{2}-1 \leqslant|f(x)|^{2}-|f(x+t)|^{2} \text {. }
$$

(iv) $|f(x+t)|>1$ and $|f(x)| \leqslant 1$. This is the same as (iii).

Next we show that

$$
|\log | \phi(x+t)|-\log | \phi(x)|| \leqslant|\log | f(x+t)|-\log | f(x)|| .
$$

We consider the same four cases as above. 
(i) In this case (13) is trivially satisfied.

(ii) In this case we have equality in (13).

(iii) $|\log | \phi(x+t)|-\log | \phi(x)||=\log |f(x)| \leqslant \log |f(x)|-\log |f(x+t)|$.

(iv) This case is the same as (iii).

Thus we have shown that $I(\phi) \leqslant I(f)$, which completes the proof of (a).

(d) Since $\|\phi\|_{\infty} \leqslant 1$ we have $\|\phi f\|_{H^{2}} \leqslant\|f\|_{H^{2}}$. Thus it will be sufficient to show that $\left\|(\phi f)^{\prime}\right\|_{B} \leqslant\left\|f^{\prime}\right\|_{B}$ (recall the definition of the $D$-norm given in (3)). Since $\phi$ is an outer function the functions $\phi f$ and $f$ have the same inner factor. Hence in computing their Dirichlet integrals by Carleson's formula the contribution from the inner factor is the same, and so if there is to be an inequality it most come from the outer factors. Thus we must show that $I(\phi f) \leqslant I(f)$ for all $x, t$. To do this we shall show that

$$
\begin{gathered}
\left.||(\phi f)(x+t)\right|^{2}-|(\phi f)(x)|^{2}|\leqslant||f(x+t)|^{2}-|f(x)|^{2} \mid \\
|\log (\phi f)(x+t)|-\log |(\phi f)(x)| \leqslant|\log | f(x+t)|-\log | f(x)|| .
\end{gathered}
$$

We consider the same four cases as in the proof of (a) above.

(i) We have $|\phi|=1$ and so equality holds in (14) and (15).

(ii) We have $|\phi f|=1$ both at $x+t$ and at $x$, and so the left side is zero in both (14) and (15),

(iii) Here $|(\phi f)(x+t)|=|f(x+t)|$ and $|(\phi f)(x)|=1$.

Then

$$
\left.||(\phi f)(x+t)\right|^{2}-\left.|(\phi f)(x)|^{2}|=1-| f(x+t)\right|^{2} \leqslant|f(x)|^{2}-|f(x+t)|^{2}
$$

which establishes (14). Also

$$
\begin{aligned}
|\log |(\phi f)(x+t)|-\log |(\phi f)(x)|| & =|\log | f(x+t)||=-\log |f(x+t)| \\
& \leqslant \log |f(x)|-\log |f(x+t)|
\end{aligned}
$$

which establishes (15).

(iv) This case is similar to (iii).

(e) We claim that for all $x, t$ we have

$$
|| \phi(x+t)|-| \phi(x)|| \leqslant|| f(x+t)|-| f(x)|| .
$$

The proof is similar to the proof of (12) above. Hence

$$
\omega(|\phi|: \delta) \leqslant \omega(|f| ; \delta) \quad(0<\delta \leqslant \pi),
$$

and so $|\phi|$ is Dini continuous. We now show that this forces $\phi$ to be continuous. This result is known but we do not have a suitable reference so we include a proof.

Let $u\left(e^{i t}\right)=\log \left|\phi\left(e^{i t}\right)\right|, 0 \leqslant t<2 \pi$, so that $u \in L^{1}(\partial \Delta)$. Let $E=\{u=-\infty\}$, i.e., $E$ is the zero set of $|\phi|$. Thus $E$ is a closed subset of $\partial \Delta$. Let $v$ denote the function conjugate to $u$; we wish to show that $v$ is continuous on $\partial \Delta \backslash E$. If $w_{0}$ is in the complement of $E$, let $J$ be a closed arc centered at $w_{0}$ and disjoint from $E$. Then $u$ is Dini continuous on $J$, and therefore $v$ is continuous at all interior points of $J$ (see [13, Corollary 1.4, Chapter III, p. 107]). Thus $e^{i v}$ is continuous and bounded on 
$\partial \Delta \backslash E$. Also, $|\phi|=e^{u}$ is continuous on $\partial \Delta$ and vanishes on $E$. Therefore $e^{u+i v}$ is continuous on $\partial \Delta$. Now take the harmonic extension of $u$ and $v$ into $\Delta$ (we denote the extended functions by $u$ and $v$ ). Since

$$
u(z)+i v(z)=\frac{1}{2 \pi} \int_{0}^{2 \pi} \frac{e^{i t}+z}{e^{i t}-z} u\left(e^{i t}\right) d t, \quad z \in \Delta,
$$

we see that $\phi=\exp (u+i v)$ in $\Delta$. Since $\phi \in H^{\infty}$ and the boundary values coincide, almost everywhere, with a continuous function on $\partial \Delta, \phi \in A$. This completes the proof of Lemma 7.

Note. V. P. Havin [14, Theorem 1] has shown how to estimate the modulus of continuity of an $f \in A$ from a knowledge of the modulus of continuity of $|f|$ on $\partial \Delta$. In particular, if $|f| \in \operatorname{Lip} \alpha$ then $f \in \operatorname{Lip}(\alpha / 2)$, and this is sharp. His work really contains (e) above, but his proof is much more complicated since he obtains a very precise result.

We wish to thank B. A. Taylor for suggesting the simple proof of (e); our original proof was more complicated.

Since $\phi$ is obtained from $f$ by a simple change it may even be true that $f \in A$ implies $\phi \in A$. This would follow if one could answer the following question in the affirmative.

Question 10. If $f \in A$ and if $\psi$ is the outer function determined by $|\psi|=\max (|f|, 1)$ on $\partial \Delta$, then must $\psi$ be in $A$ ?

Since $\phi=1 / \psi$ we see that $\phi$ and $\psi$ must both be in $A$ if either one of them is in $A$.

It is not true, however, that if $\phi \in H^{\infty}$ is an outer function and if $|\phi|$ is equal to a continuous function almost everywhere on $\partial \Delta$, then $\phi \in A$. See, for example, [17, Chapter 6, Problem 13, p. 97]. Actually, the Dini condition is sharp in some sense. This follows by taking the exponential of the examples in [13, Chapter III, Problem 4 , p. 127]. It follows from this second reference that the Dini condition cannot be improved. Indeed, if $\omega(t)$ is any increasing continuous function on $[0,2 \pi]$, vanishing at 0 , with $\omega\left(t_{1}+t_{2}\right) \leqslant \omega\left(t_{1}\right)+\omega\left(t_{2}\right)$, and if $\int_{0}^{\pi} \omega(t) t^{-1} d t=\infty$, then there is a real continuous function $u$ on $\partial \Delta$, with $\omega(u ; \delta) \leqslant \omega(\delta)$, such that the conjugate function $\tilde{u}$ is not continuous. Then the function $\phi=\exp (u+i \tilde{u})$ is an outer function in $H^{\infty}$ that is not in $A$, for which $|\phi|$ is continuous on $\partial \Delta$, with $\omega(|\phi| ; \delta) \leqslant c \omega(u ; \Delta)$ (we omit the proof).

We are now ready to give a partial answer to Question 8.

Theorem 2. If $f \in H^{\infty} \cap D$ and $g \in A \cap D$ are both cyclic, and if $|g|$ is Dini continuous on $\partial \Delta$, then $f g$ is cyclic.

Proof. By Lemma 5 it is sufficient to obtain polynomials $\left\{h_{n}\right\}$ satisfying the three conditions: (a) $\left(h_{n} g\right)(z) \rightarrow 1$ in $\Delta$, (b) $\left\|h_{n} g\right\|_{\infty} \leqslant$ const and (c) $\left\|h_{n} g\right\|_{D} \leqslant$ const.

To produce these polynomials we proceed as follows. First, since $g$ is cyclic there are polynomials $\left\{p_{n}\right\}$ such that $p_{n} g \rightarrow 1$ in $D$. We apply Lemma 7 , with $\left\{p_{n} g\right\}$ playing the role of $\left\{f_{n}\right\}$. Thus there are functions $\left\{\phi_{n}\right\}$ with $(a)^{\prime} \phi_{n}(z) \rightarrow 1$ and $\left(\phi_{n} p_{n} g\right)(z) \rightarrow 1$ in $\Delta$, (b) $\left\|\phi_{n} p_{n} g\right\|_{\infty} \leqslant$ const, and (c) $\left\|\phi_{n} p_{n} g\right\|_{D} \leqslant$ const. Also, $\left\{\phi_{n}\right\} \subset A$ since $\left|p_{n} g\right|$ is Dini continuous. Our goal now is to replace $\left\{\phi_{n}\right\}$ by a sequence of polynomials $\left\{q_{n}\right\}$ so that $(\mathrm{a})^{\prime},(\mathrm{b})^{\prime}$ and $(\mathrm{c})^{\prime}$ are still satisfied. 
Let $\left\{\sigma_{k}\left(\phi_{n} ; z\right)\right\}(k=0,1, \ldots)$ denote the Fejér means of the partial sums of the power series of $\phi_{n}(n=1,2, \ldots)$. Then $\left\|\sigma_{k}\right\|_{\infty} \leqslant\left\|\phi_{n}\right\|_{\infty}(k \geqslant 0)$ and $\left\|\sigma_{k}-\phi_{n}\right\|_{\infty} \rightarrow 0$ $(k \rightarrow \infty)$. Also, since the partial sums converge to $\phi_{n}$ in $D$, the same will be true for the sequence $\left\{\sigma_{k}\right\}$. Let $k(n)$ be a positive integer-valued function to be determined below, and let $q_{n}(z)=\sigma_{k(n)}\left(\phi_{n} ; z\right)$. We require $k(n)$ to satisfy two conditions:

1. $\left\|q_{n}-\phi_{n}\right\|_{\infty} \leqslant \min \left(n^{-1},\left\|p_{n} g\right\|_{\infty}^{-1}\right)$,

2. $\left\|q_{n}-\phi_{n}\right\|_{D} \leqslant\left\|p_{n} g\right\|_{\infty}^{-1}$.

Since $\phi_{n}(z) \rightarrow 1$ in $\Delta$, it follows from 1 that the same is true for $q_{n}$. Since $\left(p_{n} g\right)(z) \rightarrow 1$ in $\Delta$ we have $\left(q_{n} p_{n} g\right)(z) \rightarrow 1$ in $\Delta$, which establishes (a) for $\left\{q_{n}\right\}$.

Next we have

$$
\left\|q_{n} p_{n} g\right\|_{\infty} \leqslant\left\|\phi_{n} p_{n} g\right\|_{\infty}+\left\|q_{n}-\phi_{n}\right\|_{\infty}\left\|p_{n} g\right\|_{\infty} \leqslant \text { const }
$$

by (b)' and 1. This establishes (b)' for $\left\{q_{n}\right\}$.

Finally, $\left\|q_{n} p_{n} g\right\|_{D} \leqslant\left\|\phi_{n} p_{n} g\right\|_{D}+\left\|\left(q_{n}-\phi_{n}\right) p_{n} g\right\|_{D}$, and we must show that the second term on the right remains bounded. For the $H^{2}$ norm we have

$$
\left\|\left(q_{n}-\phi_{n}\right) p_{n} g\right\|_{H^{2}} \leqslant\left\|q_{n}-\phi_{n}\right\|_{\infty}\left\|p_{n} g\right\|_{H^{2}} \leqslant\left\|q_{n}-\phi_{n}\right\|_{\infty}\left\|p_{n} g\right\|_{D} \rightarrow 0 .
$$

For the Bergman norm of the derivative we have

$$
\left\|\left[\left(q_{n}-\phi_{n}\right) p_{n} g\right]^{\prime}\right\|_{B} \leqslant\left\|q_{n}-\phi_{n}\right\|_{\infty}\left\|\left(p_{n} g\right)^{\prime}\right\|_{B}+\left\|p_{n} g\right\|_{\infty}\left\|\left(q_{n}-\phi_{n}\right)^{\prime}\right\|_{B} .
$$

The first term on the right tends to zero by condition 1 since $\left\|p_{n} g\right\|_{D}$ is bounded, and the second term is bounded because of condition 2 .

If we let $h_{n}=p_{n} q_{n}$, then the $\left\{h_{n}\right\}$ are polynomials satisfying our original three conditions. This completes the proof.

Corollary 1. If $g \in A \cap D$ is cyclic and if $|g|$ is Dini continuous, then $g^{2}$ is cyclic.

Proof. Let $f=g$ in the theorem.

Corollary 2. If $g \in A \cap D$ is cyclic, if $|g|$ is Dini continuous, if $f \in D$, and if $|f(z)| \geqslant|g(z)|$ in $\Delta$, then $f$ is cyclic.

Proof. This follows from Theorem 1 and the previous corollary.

Is this last corollary contained in Corollary 1 to Theorem 1 (where it was assumed that $g \in M(D))$ ? In other words, if $g \in A \cap D$ and if $|g|$ is Dini continuous, must it be a multiplier? We think not, but have no example. Since a function satisfying a Lipschitz condition is Dini continuous, one can also consider this problem for Lipschitz functions. Proposition 12 gives some information. For more on this point see Propositions 18-20 and Question 17 in $\$ 4$.

By $\Lambda_{\alpha}$ we denote the set of those functions in $A$ that satisfy a Lipschitz condition of order $\alpha$ on $\partial \Delta$. Hardy and Littlewood showed that this is equivalent to satisfying a Lipschitz condition of order $\alpha$ in $\Delta$ (see [28] for another proof and a generalization).

By $\Lambda_{\alpha}^{2}$ we denote the subset of $H^{2}$ consisting of those functions for which the boundary values satisfy a Lipschitz condition of order $\alpha$ in the $L^{2}$-metric. Thus $\Lambda_{\alpha} \subset \Lambda_{\alpha}^{2}$. By a theorem of Hardy and Littlewood (see [11, Theorem 5.4]), if $f$ is 
holomorphic in $\Delta$ then $f \in \Lambda_{\alpha}^{2}$ if and only if

$$
M_{2}\left(f^{\prime}, r\right)=\left\{\frac{1}{2 \pi} \int_{0}^{2 \pi}\left|f^{\prime}\left(r e^{i \theta}\right)\right|^{2} d \theta\right\}^{1 / 2} \leqslant c(1-r)^{-(1-\alpha)}, \quad 0 \leqslant r<1,
$$

for $0<\alpha \leqslant 1$. Note that when $\alpha=1$ we have $\Lambda_{1}^{2}=D_{2}$. A famous theorem of $\mathrm{S}$. Bernstein states that if $\alpha>\frac{1}{2}$ then the functions in $\Lambda_{\alpha}^{2}$ have absolutely convergent power series (his result applies to $L^{2}$ as well as to $H^{2}$ ). We now show that such functions lie in $D_{1+\varepsilon}$ for suitable $\varepsilon>0$ and thus are multipliers. Part (a) of this result is related to Proposition 10(b) (see the remarks following the proof).

Proposition 12. (a) $D_{2 \alpha} \subset \Lambda_{\alpha}^{2} \subset D_{2 \beta}$ for $0<\beta<\alpha \leqslant 1$.

(b) $\Lambda_{\alpha}^{2} \subset M(D)$ for $\alpha>\frac{1}{2}$.

(c) For each $\alpha, 0<\alpha<1$, there exists $f \in \Lambda_{\alpha}$ with $f \notin D_{2 \alpha}$.

Proof. (a) Let $f(z)=\sum a_{n} z^{n} \in D_{2 \alpha}$ be given. Then

$$
\begin{aligned}
\left\{M_{2}\left(f^{\prime}, r\right)\right\}^{2} & =\sum_{1}^{\infty} n^{2}\left|a_{n}\right|^{2} r^{2 n-2}=\sum\left(n^{2-2 \alpha} r^{2 n-2}\right) n^{2 \alpha}\left|a_{n}\right|^{2} \\
& \leqslant\left\{\sup _{k} k^{2-2 \alpha} r^{2 k-2}\right\}\|f\|_{2 \alpha}^{2} .
\end{aligned}
$$

By formula (5) (preceding Proposition 10(a)) for each fixed $k$ we have

$$
(1-r)^{2 \alpha-2} \geqslant c \sum_{0}^{\infty} m^{1-2 \alpha} r^{m} \geqslant c r^{2 k} \sum_{k+1}^{2 k} m^{1-2 \alpha} \geqslant c r^{2 k} k^{2-2 \alpha} .
$$

In the last inequality we have replaced the sum by $k$ times the smallest term (the case $\alpha<\frac{1}{2}$ and $\alpha>\frac{1}{2}$ are considered separately). Combining this with the previous inequality and using (16) we see that $f \in \Lambda_{\alpha}^{2}$.

To finish the proof of (a), let $f \in \Lambda_{\alpha}^{2}$ and let $\beta<\alpha$ be given. Then $f \in D_{2 \beta}$ if and only if $f^{\prime} \in D_{2 \beta-2}$. We have, by (16),

$$
\begin{aligned}
\iint\left|f^{\prime}\left(r e^{i \theta}\right)\right|^{2}\left(1-r^{2}\right)^{1-2 \beta} r d r d \theta & =\int_{0}^{1}\left[M_{2}\left(f^{\prime}, r\right)\right]^{2}\left(1-r^{2}\right)^{1-2 \beta} r d r \\
& \leqslant c \int_{0}^{1}(1-r)^{-1+2(\alpha-\beta)} r d r<\infty .
\end{aligned}
$$

Hence by (2) we have $f \in D_{2 \beta}$.

(b) This follows from (a) since $D_{1+\varepsilon} \subset M(D)$ for $\varepsilon>0$.

(c) We construct $f$ as a gap series. Let $\alpha \in(0,1)$ be given, and let

$$
f(z)=\sum_{1}^{\infty} 2^{-n \alpha} z^{2^{n}}, \quad g(z)=(1-z)^{-1} f^{\prime}(z)=\sum A_{n} z^{n} .
$$

Then $f \notin D_{2 \alpha}$. Also, we have $M_{\infty}\left(f^{\prime}, r\right)=f^{\prime}(r)$ since the Taylor coefficients of $f^{\prime}$ are nonnegative. Since $A_{n}$ is the sum of the first $(n+1)$ Taylor coefficients of $f^{\prime}$, a calculation shows that $A_{n} \leqslant c n^{1-\alpha}$. Hence by (5), $g(r) \leqslant c(1-r)^{\alpha-2}$. Then $f^{\prime}(r) \leqslant$ $c(1-r)^{\alpha-1}$ and so $f \in \Lambda_{\alpha}$ (by the Hardy-Littlewood theorem referred to in the proof of Proposition 10(b)). This completes the proof. 
Hardy and Littlewood gave an example that would work for part (c) above. They showed that for $c>0$ the power series

$$
f(z)=\sum n^{-(1+2 \alpha) / 2} \exp (\text { icn } \log n) z^{n}
$$

represents a function in $\Lambda_{\alpha}$; clearly $f \notin D_{2 \alpha}$ (see [41, Volume I, Chapter V, Theorem 4.2]).

Proposition 10(b) said that $D_{\alpha} \subset \Lambda_{(\alpha-1) / 2}$ for $1<\alpha<3$, whereas, by Proposition 12(a), $D_{\alpha} \subset \Lambda_{\alpha / 2}^{2}$ for $0<\alpha \leqslant 2$. The latter result implies the former when $1<\alpha \leqslant 2$. This follows from three theorems of Hardy and Littlewood (see [11, Theorems 5.1, 5.4, 5.9]); we omit the details.

3. Classes of cyclic and noncyclic vectors. Up to now we have not actually identified many cyclic vectors for $D$. We know that if $|f|>c>0$, then $f$ is cyclic. But we cannot even decide, on the basis of the results obtained thus far, whether or not the function $1-z$ is cyclic. In what follows we shall use duality. Of course, since $D$ is a Hilbert space it can be regarded as being dual to itself. But it seems more fruitful to define the dual space in a different manner. We shall use the following pairing:

$$
(f, g)=\sum_{0}^{\infty} \hat{f}(n) \hat{g}(n)=\lim _{r \uparrow 1} \frac{1}{2 \pi} \int_{0}^{2 \pi} f\left(r e^{i t}\right) g\left(r e^{-i t}\right) d t,
$$

for $f \in D_{\alpha}, g \in D_{-\alpha}, \alpha \geqslant 0$. With this pairing, $D_{\alpha}$ and $D_{-\alpha}$ are dual to one another and, in particular, the Bergman space $B$ is dual to $D$.

LEMMA 8. The function $z-a$ is cyclic in $D$ if and only if $|a| \geqslant 1$.

Proof. The only new case is $|a|=1$. Suppose that we have $g \in B$ and $g \perp[(z-$ $a)$; thus $\left(g, z^{n}(z-a)\right)=0(n \geqslant 0)$. From this we have $|\hat{g}(n)|=|\hat{g}(0)|$ for all $n$. Since $\sum|\hat{g}(n)|^{2}(n+1)^{-1}<\infty$ we must have $g=0$. This completes the proof.

COROLLARY. If $p$ is a polynomial with no zeros in $\Delta$, then $p$ is cyclic.

PROOF. We have $p(z)=c\left(z-a_{1}\right) \cdots\left(z-a_{n}\right)$, where each factor is cyclic, and is a multiplier. The result now follows from Proposition 8.

Proposition 13. Let $f$ be analytic on $\Delta^{-}$with no zeros in $\Delta$. Then

(a) $f$ is cyclic in $D$, and

(b) if $\operatorname{Re} \alpha>0$ then $f^{\alpha} \in D_{1+\varepsilon}$ for $\varepsilon<2 \operatorname{Re} \alpha$, and $f^{\alpha}$ is cyclic in $D$.

Proof. (a) We have $f=p g$, where $p$ is a polynomial with no zeros in $\Delta$, and $|g|>c>0$ in $\Delta$. Then $p$ and $g$ are both cyclic and $p$ is a multiplier. The result now follows from Proposition 8.

(b) Let $(1-z)^{\alpha}=\sum a_{n}(\alpha) z^{n}$. From (5) (with $-\alpha$ replaced by $\alpha$ ) we see that $\left|a_{n}(\alpha)\right| \leqslant c(n+1)^{-1-\operatorname{Re} \alpha}$. Thus $(1-z)^{\alpha} \in D_{1+\varepsilon}$ for $\varepsilon<2 \operatorname{Re} \alpha$. Since $D_{1+\varepsilon}$ is an algebra we have $p^{\alpha} \in D_{1+\varepsilon}$, where $p$ is the polynomial from (a) above: $f=p g$. Also, $g$ has no zeros in some disk $|z|<R$ (with $R>1$ ), and therefore $g^{\alpha}$ is analytic in this disc. Hence $g^{\alpha} \in D_{1+\varepsilon}$, and so $f^{\alpha} \in D_{1+\varepsilon}$ as required. In particular, $f^{\alpha} \in M(D)$. (In the above argument we have ignored the fact that there are different possible 
determinations of $f^{\alpha}, g^{\alpha}, p^{\alpha}$, and $(1-z)^{\alpha}$ in $\Delta$. This is permissable since any two such determinations differ by a multiplicative constant.)

Finally, choose an integer $n>\operatorname{Re} \alpha$. Note that $f^{n}$ is cyclic by Proposition 8 , since $f$ is both cyclic and a multiplier. But $f^{n}=f^{\alpha} f^{n-\alpha}$ and so $f^{\alpha}$ is cyclic, by another application of Proposition 8.

Proposition 14. Let $f \in H^{\infty} \cap D$ have no zeros in $\Delta$. Assume that $f^{\alpha} \in D$ for some $\alpha>0$. If $f^{2 \beta}$ is cyclic for some $\beta \geqslant \alpha, f^{\alpha}$ is cyclic.

Proof. We may assume $|f| \leqslant 1$. Hence $\left|f^{\alpha}\right| \geqslant\left|f^{\beta}\right|$ in $\Delta$. Also, $f^{\beta} \in D$ since, with $\gamma=\beta / \alpha$,

$$
\left(f^{\beta}\right)^{\prime}=\left(\left(f^{\alpha}\right)^{\gamma}\right)^{\prime}=\gamma\left(f^{\alpha}\right)^{\gamma-1}\left(f^{\alpha}\right)^{\prime} \in B .
$$

The result now follows from Theorem 1 .

Corollary 1. Let $f \in M(D)$ be cyclic and let $\alpha>0$ be given. If $f^{\alpha} \in D$ then $f^{\alpha}$ is cyclic.

Proof. First note that $f$ has no zeros in $\Delta$ and so $f^{\alpha}$ can be defined. Also, $f^{n}$ is cyclic for all positive integers $n$, by Proposition 8 . The result now follows from the previous proposition by choosing $n>\alpha$ and letting $\beta=n$.

Corollary 2. Let $f \in A \cap D$ be cyclic, let $|f|$ be Dini continuous, and let $\alpha>0$ be given. If $f^{\alpha} \in D$ then $f^{\alpha}$ is cyclic.

Proof. We first show, by induction, that $f^{n}$ is cyclic for all positive integers $n$ (recall that $f^{n}$ is in $D$, by Lemma 4). The case $n=1$ is given. If $f^{n-1}$ is cyclic for some $n>1$ then $f^{n}=f^{n-1} f$ is cyclic by Theorem 2. Now apply Proposition 14 with $\beta=n>\alpha$.

Since the proposition and its corollaries all involve the hypothesis $f^{\alpha} \in D$ (or $M(D))$ it is of interest to see when this is satisfied.

Proposition 15. Let $f \in H^{\infty}$ have no zeros in $\Delta$, and let $\alpha \geqslant 1$ be given. If $f \in D$ then $f^{\alpha} \in D$; if $f \in M(D)$ then $f^{\alpha} \in M(D)$.

Proof. $\left(f^{\alpha}\right)^{\prime}=\alpha f^{\alpha-1} f^{\prime} \in B$ since $f^{\alpha-1}$ is bounded; thus $f^{\alpha} \in D$. For the second part, let $g \in D$ be given. Then $\left(f^{\alpha} g\right)^{\prime}=f^{\alpha} g^{\prime}+\alpha f^{\alpha-1} f^{\prime} g$. Also, $f^{\prime} g \in B$ since $f$ is a multiplier. Thus $\left(f^{\alpha} g\right)^{\prime} \in B$, as required.

We now present an example to show that, in general, if $\alpha<1$ then $f^{\alpha}$ need not be in $D$, even when $f \in M(D)$.

Proposition 16. Let

$$
f(z)=f_{\beta, a}(z)=(1-z)^{\beta} \exp \left\{a \frac{z+1}{z-1}\right\}, \quad a>0, \beta>0 .
$$

If $\beta>\frac{1}{2}$, then $f \in M(D)$ for all $a>0$. If $\beta \leqslant \frac{1}{2}$, then $f \notin D$ for any $a>0$.

Proof. Let

$$
g(z)=-(1-z)^{\beta-2} \exp \left\{a \frac{z+1}{z-1}\right\}
$$


Then $f^{\prime}(z)=[\beta(1-z)+2 a] g(z)$. We have

$$
2 a \leqslant|\beta(1-z)+2 a| \leqslant 2 a+2 \beta, \quad|z|<1 .
$$

First suppose that $\beta \leqslant \frac{1}{2}$. To show that $f \notin D$ we must show that $g \notin B$. To simplify the calculations we map to the right half-plane. Let

$$
w=-\frac{z+1}{z-1}, \quad z=\phi(w)=\frac{w-1}{w+1} .
$$

If $z=x+i y, w=u+i v$ then

$$
\begin{aligned}
\iint_{\Delta}|g(z)|^{2} d x d y & =\iint_{u>0}|g(\phi(w))|^{2}\left|\phi^{\prime}(w)\right|^{2} d u d v \\
& =c \int_{0}^{\infty} e^{-2 a u}\left(\int_{-\infty}^{\infty}|w+1|^{-2 \beta} d v\right) d u
\end{aligned}
$$

But this is infinite since for each $u \geqslant 0$ we have

$$
\int_{-\infty}^{\infty}|w+1|^{-2 \beta} d v \geqslant \int_{u+1}^{\infty}\left[(u+1)^{2}+v^{2}\right]^{-\beta} d v \geqslant \int_{u+1}^{\infty}\left(2 v^{2}\right)^{-\beta} d v=\infty
$$

because $\beta \leqslant \frac{1}{2}$. Thus $\mathrm{f} \notin D$, as was to be shown.

One could use similar calculations to show that $f \in D$ when $\beta>\frac{1}{2}$. However, we wish to show that $f \in M(D)$. Since $f$ is bounded this is equivalent to showing that $f^{\prime} h \in B$ for all $h \in D$. By (18) this is equivalent to showing that $g h \in B$. Without loss of generality we may assume that $h(0)=0$. Hence $|h(z)|^{2} \leqslant-c \log \left(1-|z|^{2}\right)$ (this follows from the Cauchy inequality). Recall that we use $c$ to denote a general constant, not necessarily the same at each occurrence. We have

$$
\begin{aligned}
\iint_{\Delta}|g h|^{2} d x d y & \leqslant c \iint|g(z)|^{2} \log \frac{1}{1-|z|^{2}} d x d y \\
& =c \int_{0}^{\infty} e^{-2 a u}\left(\int_{-\infty}^{\infty}\left[(u+1)^{2}+v^{2}\right]^{-\beta} \log \frac{(u+1)^{2}+v^{2}}{4 u} d v\right) d u \\
& =c \int_{0}^{\infty} e^{-2 a u} I(u) d u .
\end{aligned}
$$

Thus

$$
I(u)=2 \int_{0}^{\infty}=2\left(\int_{0}^{u+1}+\int_{u+1}^{\infty}\right)=2\left(I_{1}+I_{2}\right)
$$

We have

$$
\begin{aligned}
I_{1}(u) & \leqslant \int_{0}^{u+1}(u+1)^{-2 \beta} \log \frac{2(u+1)^{2}}{4 u} d v \leqslant(u+1)^{1-2 \beta} \log \frac{(u+1)^{2}}{u} \\
& \leqslant 2 \log (u+1)-\log u .
\end{aligned}
$$

Choose $\varepsilon>0$ so that $2 \beta-\varepsilon>1$. Then $\log v^{2} \leqslant c v^{\varepsilon}$ for $v \geqslant 1$, and so

$$
\begin{aligned}
I_{2}(u) & \leqslant \int_{u+1}^{\infty} v^{-2 \beta} \log \frac{2 v^{2}}{4 u} d v \leqslant \int_{u+1}^{\infty}\left(c v^{\varepsilon-2 \beta}-v^{-2 \beta} \log 2 u\right) d v \\
& \leqslant c+c|\log 2 u| .
\end{aligned}
$$

Therefore $\int_{0}^{\infty} e^{-2 a u} I(u) d u<\infty$, which completes the proof. 
In this example $f$ has an inner factor. Question: if $f \in D$ is outer, must $f^{1 / 2} \in D$ ? Note that, by Proposition 15, an affirmative answer to this question would imply that $f^{\alpha} \in D$ for $0<\alpha<1$. However, this is probably not correct. An example could perhaps be built up using Proposition 16. Namely, let

$$
g_{n}=\exp \left\{a_{n}\left(r_{n} z+1\right) /\left(r_{n} z-1\right)\right\},
$$

where $a_{n} \downarrow 0$ and $r_{n} \uparrow 1$ rapidly. Then $h=g_{1} g_{2} \cdots$ is an outer function, and $f=(1-z) h$ should be the desired example. However, we have not verified the details.

Next we state some results and introduce some notation that will be needed for our next two theorems.

1. A closed subset $J$ of a separable metric space is uniquely expressible as the disjoint union of a countable set and a perfect set (which we shall call the "perfect core" of $J$ ). By a perfect set we mean a set that is closed and has no isolated points. Note that, with this definition, the empty set is perfect.

2. We recall the "thin sets" in the sense of Beurling, Carleson and Hayman that were introduced in the discussion following equation (4). We need the following facts about these sets. First, every closed subset of a thin set is thin. Second, if $f \in D_{2}$ then the boundary zero set of $f$ is a thin set. (Actually this is true whenever $f \in \operatorname{Lip} \varepsilon$ for $\varepsilon>0$, and by Proposition 10(b) this applies to $D_{\alpha}$ for $\alpha>1$.) Finally, if $K$ is a thin set then there exists an outer function $\phi \in A^{\infty}$ (that is, $\phi$ and all its derivatives are in the disc algebra), such that $\phi$ and all its derivatives vanish on $K$, but $\phi$ has no other zeros in $\Delta^{-}$(see $\left.[8, \S \mathrm{I} ; 20]\right)$.

3. Notation. (a) If $f \in D$, then

$$
Z(f)=\left\{e^{i t}: \lim f\left(r e^{i t}\right)=0, r \uparrow 1\right\} .
$$

(b) If $S \subset D$ is any subset, then [ $S$ ] denotes the smallest closed subspace of $D$ that contains $S$ and is invariant under multiplication by $z$. This is consistent with our earlier notation $[f]$ for $f \in D$. Also, note that $[[S]]=[S]$.

(c) If $K \subset \partial \Delta$ is compact, let $I(K)=\left\{f \in D_{2}: f=0\right.$ on $\left.K\right\}$. This is a closed ideal in $D_{2}$; it contains just the zero function if and only if $K$ is not a thin set (see 2 above).

4. If $S \subset D$ is a closed invariant subspace (for multiplication by $z$ ), then $S \cap D_{2}$ is a closed ideal in $D_{2}$.

Lemma 9. If $K \subset \partial \Delta$ is closed, $K_{0}=K \cup\left\{a_{0}\right\}$ where $a_{0} \in \partial \Delta \backslash K$, if $I_{0}=I\left(K_{0}\right)$ and $I=I(K)$, then $\left[I_{0}\right]=[I]$.

Proof. Since $I_{0} \subset I$ we have $\left[I_{0}\right] \subset[I]$. To prove the reverse containment it is enough to show that $I \subset\left[I_{0}\right]$. If this were false, then there would be an $f \in I \backslash\left[I_{0}\right]$. We may assume that $f\left(a_{0}\right)=1$. Then there is a continuous linear functional $\lambda$ on $D$ such that $\lambda=0$ on $\left[I_{0}\right]$ but $(f, \lambda)=1$. We claim that if $g$ is any element in $I$ with $g\left(a_{0}\right)=1$, then $(g, \lambda)=1$. Indeed, $f-g \in I_{0}$ and so $(f-g, \lambda)=0$.

Since point evaluation at $a_{0}$ is not a bounded linear functional on $D$, there exist polynomials $\left\{p_{n}\right\}$ such that $p_{n}\left(a_{0}\right)=1$ for all $n$ but $\left\|p_{n}\right\| \rightarrow 0$. Then $p_{n} f \in I$ and 
$\left(p_{n} f\right)\left(a_{0}\right)=1$; therefore $\left(p_{n} f, \lambda\right)=1$. But this is a contradiction since $p_{n} f \rightarrow 0$ in $D$ (recall that the elements of $D_{2}$ are multipliers on $D$ ).

THEOREM 3. If $f \in D_{2}$ is an outer function and if $f$ has at most countably many zeros on $\partial \Delta$, then $f$ is cyclic in $D$.

This theorem is a special case of the following (take $g=1$ )

THEOREM 4. If $f, g \in D_{2}$ are outer functions, and if $Z(f)$ and $Z(g)$ have the same perfect core, then $[f]=[g]$.

Proof. We work first with $f$. Let $I_{f}=[f] \cap D_{2}$. By 4 above this is a closed ideal in $D_{2}$ that contains $f$. It follows from the Main Theorem in B. I. Korenblum's paper [21, Introduction] that a closed ideal in $D_{2}$ that contains an outer function must have the form $I(K)$ for some closed $K \subset \partial \Delta ; K$ is necessarily a thin set. Thus there is a thin set $K_{f}$ such that $I_{f}=I\left(K_{f}\right)$; also, $K_{f} \subset Z(f)$.

We claim that $K_{f}$ is a perfect set. Indeed, assume that $K_{f}$ contained an isolated point $a_{0}$. Let $K=K_{f} \backslash\left\{a_{0}\right\}$ and $I=I(K)$. By Lemma 9, $[I]=\left[I_{f}\right]$. Also, since $K$ is thin there is an $h \in D_{2}$ such that $h=0$ on $K$ and $h\left(a_{0}\right)=1$. Thus $h \in I \subset[I]=\left[I_{f}\right]$. Note that since $f \in I_{f}=[f] \cap D_{2} \subset[f]$ we have

$$
[f] \subset\left[I_{f}\right] \subset[[f]]=[f]
$$

and therefore $\left[I_{f}\right]=[f]$. Hence $h \in[f] \cap D_{2}=I_{f}$. But this is a contradiction since $h\left(a_{0}\right)=1$. Thus, $K_{f}$ is a perfect set, and so $K_{f}$ is contained in the perfect core of $Z(f)$.

If we repeat the above argument for $g$ we see that $K_{g}$ is contained in the perfect core of $Z(g)$. Since these two perfect cores are equal we see that $f=0$ on $K_{g}$ and thus $f \in I_{g}=[g] \cap D_{2}$. Thus $f \in[g]$ and, similarly, $g \in[f]$, which completes the proof.

Thus we see that for an outer function $f$ in $D_{2}$, cyclicity depends only on the zero set $Z(f)$, and in fact, only on the perfect core of this set. We now show that if $f$ has too large a zero set then $f$ is not cyclic. The facts about logarithmic capacity that we need may all be found, for example, in Chapter III of [39]. We shall use "hats" to denote the Fourier coefficients of functions and of measures:

$$
\hat{h}(n)=\int \bar{w}^{n} h(w) d m(w), \quad \hat{\mu}(n)=\int \bar{w}^{n} d \mu(w) \quad(n=0, \pm 1, \pm 2, \ldots),
$$

where $h \in L^{1}(\partial \Delta), d m$ denotes normalized Lebesgue measure on $\partial \Delta, \mu$ is a complexvalued Borel measure on $\partial \Delta$, and $|w|=1$.

THEOREM 5. If $f \in D$ and if $Z(f)$ has positive logarithmic capacity, then $f$ is not cyclic.

Proof. Let $J_{n}=\left\{e^{i \theta} \in Z(f):\left|f\left(r e^{i \theta}\right)\right| \leqslant n, 0 \leqslant r<1\right\}, n=1,2, \ldots$. These are Borel sets and $Z(f)=\bigcup J_{n}$. Since a countable union of Borel sets of capacity zero must have capacity zero (see [39, Theorem III 8, p. 57]), there exists an integer $N$ such that $J_{N}$ has positive capacity. Hence there is a compact set $F \subset J_{N}$ and a Borel 
probability measure $\mu$ supported in $F$ with finite energy integral. This means that

$$
I(\mu)=\iint \log \frac{1}{|z-w|} d \mu(z) d \mu(w)<\infty .
$$

Let $k_{w}=k_{w}(z)=(1-w z)^{-1}$. Then $k_{2} \in D_{-2}$ for $|w|=1$, and the map $w \mapsto k_{w}$ is a norm continuous map of $\partial \Delta$ into $D_{-2}$. Hence the vector-valued integral, $g=\int k_{w} d \mu(w)$, exists and defines an element of $D_{-2}$. Our goal is to show that $g \in B, g \neq 0$, and $(p f, g)=0$ for all polynomials $p$. This will prove the theorem since $B$ may be identified with the dual space of $D$ by means of the pairing (17).

We first find a formula for the value of $g$ at a given point $z \in \Delta$, and then we find the power series for $g$. Thus let $z$ denote a fixed point in $\Delta$, and let $\lambda_{z}$ denote the linear functional on $D_{-2}$ of evaluation at $z$. Then we have

$$
\begin{aligned}
g(z) & =\lambda_{z}\left(\int k_{w} d \mu(w)\right)=\int \lambda_{z}\left(k_{w}\right) d \mu(w)=\int(1-w z)^{-1} d \mu(w) \\
& =\int\left(\sum_{0}^{\infty} w^{n} z^{n}\right) d \mu(w)=\sum_{0}^{\infty}\left(\int w^{n} d \mu(w)\right) z^{n}=\sum_{0}^{\infty} \hat{\mu}(-n) z^{n},
\end{aligned}
$$

since the series converges uniformly in $w(|w|=1)$. Also, $g \neq \equiv$, otherwise $\hat{\mu}(n)=0$ $(n \leqslant 0)$. But then $\hat{\mu}(n)=0$ for all $n(\hat{\mu}(-n)=\overline{\hat{\mu}(n)}$ since $\mu$ is a real measure) which is impossible.

Next we show that $g \in B$. Let $h(t)=-\log \left|1-e^{i t}\right|$. Then one sees that, in (19), $I(\mu)=\iint h(\theta-t) d \mu\left(e^{i t}\right) d \mu\left(e^{i \theta}\right)$. Since $h$ is an even periodic function, and is convex for $0<t<2 \pi$, we have

$$
I(\mu)=\sum_{-\infty}^{\infty} \hat{h}(n)|\hat{\mu}(n)|^{2}
$$

by Proposition 3 of [18] (see p. 35). (Strictly speaking they require, in addition, that $h \geqslant 0$. However, this is only used to obtain $\hat{h}(0) \geqslant 0$, and in the present case, as we shall see, $\hat{h}(0)=0$. Alternatively, if $c$ is a large positive number then $h+c \geqslant 0$ and their result can be applied.) To find the Fourier series of $h$ we proceed as follows:

$$
h(t)=\frac{1}{2}\left\{-\log \left(1-e^{i t}\right)-\log \left(1-e^{-i t}\right)\right\}=\frac{1}{2}\left\{\sum_{1}^{\infty} \frac{e^{i n t}}{n}+\sum_{1}^{\infty} \frac{e^{-i n t}}{n}\right\} .
$$

Thus $\hat{h}(0)=0$, and $\hat{h}(n)=1 / 2|n|$ for $n \neq 0$. Also, $|\hat{\mu}(-n)|=|\hat{\mu}(n)|$ since $\mu$ is a real measure, and so

$$
I(\mu)=\sum_{1}^{\infty} \frac{1}{n}|\hat{\mu}(n)|^{2}<\infty .
$$

Using this result together with the power series for $g$ from (20), we see that $g \in B$.

It remains to show that $(p f, g)=0$ for all polynomials $p$. Let $d m$ denote normalized Lebesgue measure on the circle; the limits below are taken as $r \uparrow 1$. From 
(17) and (20) we have

$$
\begin{aligned}
\langle p f, g\rangle & =\lim \int(p f)\left(r e^{i t}\right) g\left(r e^{-i t}\right) d m(t) \\
& =\lim \int(p f)\left(r e^{i t}\right) \int\left(1-w r e^{-i t}\right)^{-1} d \mu(w) d m(t) \\
& =\lim \iint(p f)\left(r e^{i t}\right)\left(1-w r e^{-i t}\right)^{-1} d m(t) d \mu(w) \\
& =\lim \int_{F}(p f)\left(r^{2} w\right) d \mu(w)=0,
\end{aligned}
$$

by the bounded convergence theorem. This completes the proof.

Beurling [4] has shown that if $f \in D$, then the set of $e^{i \theta}$ for which a finite radial limit does not exist is a set of logarithmic capacity zero. From this we see that if $Z(f)$ has positive capacity then $1 / f$ is not in $D$, and therefore one cannot hope to find a negative example for Question 4 from Theorem 5. Carleson has shown that for bounded functions in $D$ a stronger result is true: the set where a finite radial limit does not exist has logarithmic length zero (see [7, Chapter III, §3]).

If $E$ is a Borel subset of $\partial \Delta$ of positive capacity, then we define

$$
D_{E}=\left\{f \in D: \lim _{r \uparrow 1} f\left(r e^{i \theta}\right)=0 \text {, a.e.-cap, in } E\right\},
$$

where "a.e.-cap" means "except for a set of capacity zero".

Carleson [8, pp. 332-335] has introduced a class of thin sets $E$ having positive capacity, with the property that $\left\{f \in D_{E}: f(0)=1\right\}$ is a closed subset of $D$ (see Theorem 6, p. 335 in his paper). From this it follows easily that $D_{E}$ itself is a closed set (and therefore any function in $D_{E}$ is noncyclic).

Joel Shapiro has pointed out to us that $D_{E}$ is always a closed subspace of $D$, for any $E \subset \partial \Delta$. This follows from results in [24]. More specifically, we use their Theorem 3.12, inequality (2), with $p=2$ and $K\left(e^{i \theta}\right)=\left|1-e^{i \theta}\right|^{-1 / 2}$. Then their space $K * L^{2}$ coincides with $D$, and, what is more difficult, their capacity is equivalent to logarithmic capacity. We obtain the following inequality ("cap" denotes "logarithmic capacity"):

$$
\operatorname{cap}\left\{e^{i \theta}:\left|f\left(e^{i \theta}\right)\right|>\lambda\right\} \leqslant c(\|f\| / \lambda)^{2}, \quad f \in D .
$$

To show that $D_{E}$ is closed we assume that $\left\{f_{n}\right\} \subset D_{E}$ and $f_{n} \rightarrow f$ in $D$. Then for each $n$ we have

$$
\begin{aligned}
\operatorname{cap}\left\{e^{i \theta} \in E:|f|>\lambda\right\} & =\operatorname{cap}\left\{e^{i \theta} \in E:\left|f_{n}-f\right|>\lambda\right\} \\
& \leqslant \operatorname{cap}\left\{e^{i \theta} \in \partial \Delta:\left|f_{n}-f\right|>\lambda\right\} \leqslant c\left(\left\|f_{n}-f\right\| / \lambda\right)^{2} .
\end{aligned}
$$

Letting $n \rightarrow \infty$ we have $\operatorname{cap}\left\{e^{i \theta} \in E:|f|>\lambda\right\}=0$, for all $\lambda>0$. If we take $\lambda=1 / k(k=1,2, \ldots)$ and use the fact that a countable union of sets of capacity 0 has capacity 0 , then we see that $\operatorname{cap}\left\{e^{i \theta} \in E:|f|>0\right\}=0$, that is, $f \in D_{E}$. Of course our Theorem 5 is an immediate corollary of this (our proof is entirely different and is a good deal simpler). 
Question 11. If $f \in D$ is an outer function, then does [ $f]=D_{E}$ for some $E \subset \partial \Delta$ ?

A comparison of Theorems 3, 4 and 5 suggests that, for outer functions $f$ in $D$, the set $Z(f)$ determines whether or not $f$ is cyclic. In particular, Theorem 4 says that this is indeed the case when, in addition, $f \in D_{2}$. We conjecture that the converse to Theorem 5 is valid, that is, that an outer function $f \in D$ is cyclic if and only if $Z(f)$ has logarithmic capacity 0.

Question 12. If $f \in D$ is outer and if $Z(f)$ has logarithmic capacity 0 , then must $f$ be cyclic?

If our conjecture is correct, then this would immediately imply an affirmative answer to Question 3, and would also yield an affirmative answer to Question 4. Indeed, if $f$ and $1 / f$ are both in $D$ then, since the radial limit exists and is finite except perhaps on a set of capacity 0 (see [4]), we see that $Z(f)$ and $Z(1 / f)$ must both have capacity 0 .

At present, however, we have no example of cyclic $f$ for which $Z(f)$ is uncountable, although univalent functions may provide such examples.

A univalent function $f$ is in $D$ if and only if it maps the unit disc onto a plane region $G$ of finite area. We distinguish three cases.

1. If $0 \in G$ then $f$ is not cyclic.

2. If $0 \notin G^{-}$then $|f| \geqslant c>0$ in $\Delta$ and so $f$ is cyclic.

3. The case $0 \in \partial G$ is open.

We first show that cyclicity depends only on the region $G$ and not on the particular mapping $f$ of $\Delta$ onto $G$.

Lemma 10. Let $f \in D$ and let $\phi$ be a bilinear map of $\Delta$ onto itself. Then (1) $f \circ \phi \in D$, and (2) $f \circ \phi$ is cyclic if and only if $f$ is cyclic.

Proof. We have $\left\|(f \circ \phi)^{\prime}\right\|_{B}=\left\|f^{\prime}\right\|_{B}$ (this may be seen by changing variables in the double integral). Also, composition with $\phi$ (or with any inner function) is a bounded operator on $H^{2}$, with bound $(1+|\alpha|) /(1-|\alpha|)$, where $\alpha=\phi(0)$ (see [29]). Thus by formula (3) for the norm in $D$ we see that composition with $\phi$ is a bounded linear transformation on $D$. Hence if there are polynomials $\left\{p_{n}\right\}$ such that $p_{n} f \rightarrow 1$ in $D$, then $\left(p_{n} \circ \phi\right)(f \circ \phi)=\left(p_{n} f\right) \circ \phi \rightarrow 1$ also. But for each $n, p_{n} \circ \phi \in M(D)$ since it is analytic on $\Delta^{-}$. Thus $f \circ \phi$ is also cyclic. The converse follows since the bilinear maps form a group under composition.

We pause to record another problem. If $\phi$ is analytic in $\Delta$ with $\phi(\Delta) \subset \Delta$ then we let $C_{\phi} f=f \circ \phi$, for all $f$ analytic in $\Delta$.

Question 13. For which $\phi$ is $C_{\phi}$ a bounded operator (a compact operator) on $D$ ?

In the proof of the lemma we showed that $C_{\phi}$ is bounded (and invertible) when $\phi$ is a bilinear map. It is easy to see that $C_{\phi}$ is compact if $\phi(\Delta)^{-} \subset \Delta$; it can be shown that $C_{\phi}$ is a Hilbert-Schmidt operator if and only if $\iint(1-|\phi|)^{-2}\left|\phi^{\prime}\right|^{2}<\infty$. For a survey of results in the $H^{2}$ case see Nordgren [25]. For some results on the problem for $D_{2}$ (and related spaces) see [27].

To return to univalent functions we recall two results. First, a nonvanishing univalent function in the unit disc must be an outer function (see [11, Theorem 3.17]). Second, Beurling [4] showed that if $f$ is univalent and has a finite Dirichlet 
integral (i.e., $f \in D$ ), then its boundary zero set (i.e., $Z(f)$ ) has logarithmic capacity zero. In view of Question 12 this suggests the following problem.

Question 14. If $f \in D$ is a nonvanishing univalent function, must it be cyclic?

We give an affirmative answer for the special case when $g$ is the slit disc (that is, $G$ is formed from the open unit disc by deleting the unit interval).

Proposition 17. If $f$ is any conformal map of $\Delta$ onto the slit disc, then $f$ is cyclic.

Proof. Let $g(z)=1+z+i(1-z), h(z)=2\left\{i\left(1-z^{2}\right)\right\}^{1 / 2}$. Then it can be shown that $f=(g-h) /(g+h)$ is one such map, with $f(i)=0$. (First, map to the upper half-plane with 0 going to $i$; then use the square root map to go to the first quadrant; next use the inverse of the first map to go to the upper half-disc; then square. We use the principal branch of the square root function defined in the plane slit along the negative real axis.) We note in passing that by considering the Taylor coefficients one can show that $f \in D_{\alpha}$ for all $\alpha<2$, but $f \notin D_{2}$.

The functions $g-h$ and $g+h$ are both in $H^{\infty} \cap D$ and their product is cyclic since it is a polynomial with no zeros in $\Delta$ (see the Corollary to Lemma 8 ); therefore both $g-h$ and $g+h$ are cyclic (Proposition 11). Therefore $(g+h) f$ is cyclic and so, again by Proposition $11, f$ is cyclic.

We do not know whether the conclusion remains valid when the standard slit disc is replaced by the disc with a "fat" slit (that is, from $\Delta$ remove a Jordan arc of positive two-dimensional measure going from 0 to 1 in $\Delta$ ).

4. Miscellaneous results. We now return to Question 2: is Axiom 7 independent of the six original axioms? Here we are dealing with a general Banach space of analytic functions in a bounded region $G$. Recall that an equivalent statement to Axiom 7 is the following: $M(E) f \subset[f]$, for all $f \in E$. We rewrite this as follows ( $P$ denotes the set of all polynomials):

$$
\forall \phi \in M(E), \forall f \in E, \forall \varepsilon>0, \exists p \in P \text { such that }\|p f-\phi f\|_{E}<\varepsilon .
$$

A sufficient condition for this is that $P$ be dense in $M(E)$ in the strong operator topology (this was how Proposition 7 was proved). This means the following:

$$
\begin{aligned}
& \forall \phi \in M(E), \forall F \subset E \text { (finite subset), } \forall \varepsilon>0, \\
& \exists p \in P \text { such that }\|p f-\phi f\|_{E}<\varepsilon \text { for all } f \in F .
\end{aligned}
$$

We write SOT to denote the strong operator topology.

We now present a class of examples where the first six axioms hold. It seems likely that Axiom 7 will not always hold.

We begin with the open unit disc $\Delta$. Let $K$ be a compact convex set with nonempty interior, such that $1 \in K$ and $K \subset \Delta \cup\{1\}$. Let $G=\Delta \backslash K$, and let $E=L_{a}^{2}(G)$, that is, the space of area square-integrable analytic functions in $G$ (the "Bergman space" of $G$ ). It is known that for a suitable choice of $K$ (but not for all choices), $P$ will be dense in $E$. (See the 1953 survey article [23] by Mergelyan; for more recent results see, for example, [6].) Thus $E$ satisfies the first six axioms. Also, $M(E)=H^{\infty}(G)$ (and the operator norm coincides with the supremum norm). 
If one could show that $P$ were not SOT dense in $H^{\infty}(G)$, then this would be evidence suggesting that Axiom 7 might not hold. In this direction we can only prove the following: $P$ is not sequentially SOT dense in $H^{\infty}(G)$. Indeed, let $\alpha$ be an interior point of $K$ and let $\phi(z)=(z-\alpha)^{-1}$. Then $\phi \in H^{\infty}(G)$. We will show that there is no sequence $\left\{p_{n}\right\}$ of polynomials such that $p_{n} \rightarrow \phi$ SOT. Suppose that such a sequence did exist. Then by the principle of uniform boundedness, $\left\{p_{n}\right\}$ would be uniformly bounded in $G$. But then by the maximum principle, $\left\{p_{n}\right\}$ would be uniformly bounded in $\Delta$ and thus would form a normal family. Hence a subsequence converges, uniformly on compact subsets of $\Delta$, to a function $g \in H^{\infty}(\Delta)$. But $p_{n}(z) \rightarrow \phi(z)$ for $z \in G$. Therefore, $g$ is an analytic continuation of $\phi$, which is impossible.

To show that $P$ is not SOT dense it would suffice to show that the weak operator topology (WOT), restricted to $H^{\infty}(G)$, coincides with the weak* topology (which $H^{\infty}(G)$ inherits from $L^{\infty}(G)$ ). Indeed, the restriction map $f \mapsto f \mid G$ is an isometric mapping of $H^{\infty}(\Delta)$ into $H^{\infty}(G)$. It follows that $H^{\infty}(\Delta) \mid G$ is weak* sequentially closed in $H^{\infty}(G)$. Now by a theorem of Banach [3, Chapitre VII, Théorème 5]) it follows that $H^{\infty}(\Delta) \mid G$ is weak* closed. Thus (if these topologies coincide) it is WOT closed, and hence is SOT closed (see [10, V1.1.5, p. 447]). Thus the SOT closure of $P$ would be equal to $H^{\infty}(\Delta) \mid G$. To state the topological problem more explicitly we note that if $\left\{\phi_{\alpha}\right\} \subset H^{\infty}$ is a net, then $\phi_{\alpha} \rightarrow 0$ (WOT) means

$$
\iint_{G} \phi_{\alpha} f \bar{g} d x d y \rightarrow 0 \quad\left(f \in L_{\alpha}^{2}(G), g \in L^{2}(G)\right)
$$

( $g$ need not be analytic), whereas $\phi_{\alpha} \rightarrow 0$ (weak*) means

$$
\iint_{G} \phi_{\alpha} h d x d y \rightarrow 0 \quad\left(h \in L^{1}(G)\right) .
$$

Next we pose two more questions about the space $D$. The first is related to Question 11; an affirmative answer would simplify the problem of classifying the invariant subspaces of the operator $M_{z}$ on the space $D$.

Question 15. If $F \subset D$ is a closed subspace invariant under the operator $M_{z}$ (i.e., $z F \subset F$ ), then must there exist $f \in D$ with $F=[f]$ ?

Question 16. If $f \in D$, must there exist $\phi_{1}, \phi_{2} \in M(D)$ such that $f=\phi_{1} / \phi_{2}$ ?

This would be an analogue of the result for $H^{2}$, where each function is the quotient of two bounded functions. Such a result is not valid in $D_{\alpha}$ for $\alpha<0$ since $M\left(D_{\alpha}\right)=H^{\infty}$, whereas $D_{\alpha}$ contains functions not in the Nevanlinna class. On the other hand for $\alpha>1$ the result is trivially true since $M\left(D_{\alpha}\right)=D_{\alpha}$, indeed, $f=f / 1$. However it seems likely that the answer to Question 16 is negative. Indeed, an affirmative answer would imply, in particular, that every function in $D$ is the quotient of two bounded functions in $D$. And Carleson has indicated that this is probably not correct (see [7, Chapter III, §3; especially p. 39]); see the remarks on radial limits following the proof of Theorem 5 .

An affirmative answer to Question 16 would be interesting in operator theory. An algebra of operators on a Banach space is said to be transitive if there is no proper closed subspace mapped into itself by all operators in the algebra. In [2] Arveson 
showed that if a transitive algebra of operators on $H^{2}$ contains the operator of multiplication by $z$ (the unilateral shift operator), then the algebra is SOT dense in the algebra of all operators. It has been an open question if the conclusion remains valid when the unilateral shift is replaced by a weighted shift. Arveson's proof depended on the fact that $H^{2}$ functions are quotients of bounded functions and so, if Question 16 could be answered in the affirmative, then the unilateral shift could be replaced by the operator of multiplication by $z$ on the space $D$ in his theorem.

We now prove three propositions about multipliers on $D$.

Proposition 18. (a) If $\phi \in M(D)$ then

$$
\left|\phi^{\prime}(z)\right|^{2} \leqslant c \frac{r}{(1-r)^{2} \log (1 /(1-r))} \quad(r=|z|) .
$$

(b) If $\phi \in H^{\infty}$ and $\sum(n \log n)|\hat{\phi}(n)|^{2}<\infty$, then $\phi \in M(D)$.

Proof. (a) This is contained in Theorem 1 of [38].

(b) As observed earlier, if $\phi \in H^{\infty}$ then $\phi \in M(D)$ if and only if $\phi^{\prime} f \in B$ for all $f \in D$ (see remark 7 following Proposition 3).

We first show that if $\phi \in H^{\infty}$ and if

$$
M_{2}\left(\phi^{\prime}, r\right)\left(\log \frac{1}{1-r}\right)^{1 / 2} \in L^{2}(0,1),
$$

then $\phi \in M(D)$. Indeed, let $f \in D$. From the Cauchy(-Buniakovsky-Schwarz) inequality we have

Therefore

$$
|f(z)|^{2} \leqslant\left(\frac{1}{r} \log \frac{1}{1-r}\right)\|f\|^{2} \quad(r=|z|) .
$$

$$
\iint\left|\phi^{\prime} f\right|^{2} r d r d \theta \leqslant c \int_{0}^{1}\left(M_{2}\left(\phi^{\prime}, r\right)\right)^{2} \log \frac{1}{1-r} d r<\infty,
$$

and so $\phi^{\prime} f \in B$ as required.

We now show that (23) is equivalent to the convergence of the series in (b). Indeed if $\phi=\sum a_{n} z^{n}$ then

$$
\int_{0}^{1} M_{2}\left(\phi^{\prime}, r\right)^{2} \log \frac{1}{1-r} d r=\sum_{1}^{\infty} n^{2}\left|a_{n}\right|^{2} \int_{0}^{1} r^{2 n-2} \log \frac{1}{1-r} d r
$$

If $r_{n}=1-1 / n$, then $r^{2 n-2} \geqslant c$ in $\left[r_{n}, 1\right)$. Thus

$$
\int_{0}^{1} r^{2 n-2} \log \frac{1}{1-r} d r \geqslant c \int_{r_{n}}^{1} \log \frac{1}{1-r} d r \geqslant c \frac{\log n}{n} .
$$

On the other hand,

$$
\begin{gathered}
\int_{0}^{1} r^{2 n-2} \log \frac{1}{1-r} d r=\int_{0}^{r_{n}}+\int_{r_{n}}^{1}=\mathrm{I}_{n}+\mathrm{II}_{n}, \\
\mathrm{I}_{n} \leqslant(\log n) \int_{0}^{r_{n}} r^{2 n-2} d r \leqslant c \frac{\log n}{n}, \\
\mathrm{II}_{n} \leqslant \int_{r_{n}}^{1} \log \frac{1}{1-r} d r=\frac{1+\log n}{n} .
\end{gathered}
$$

This completes the proof of (b). 
(b) above may be compared with a result of G. D. Taylor: if $w_{n} \uparrow \infty, \Sigma\left(n w_{n}\right)^{-1}<$ $\infty, \sum n w_{n}\left|a_{n}\right|^{2}<\infty$, then $\phi=\sum a_{n} z^{n} \in M(D)$. (This follows from Theorem 5 of [38] if one takes $n_{k}=k$.)

Of course the convergence of the series in (b) above does not imply $\phi \in H^{\infty}$. However, if one puts in random \pm 1 in the power series then almost surely the series will converge uniformly and therefore will be in the disc algebra. This follows from a result of Paley and Zygmund [26] (see Theorem VII, p. 347 and Theorem XVIII, p. 461): if $\sum\left|a_{n}\right|^{2}(\log n)^{1+\varepsilon}<\infty$, then $\sum \pm a_{n} z^{n}$ converges uniformly, almost surely. Thus there are functions that are in the disc algebra, and in $M(D)$, that do not have absolutely convergent power series. This contrasts with the example mentioned earlier where $a_{n} \downarrow 0, \sum a_{n}<\infty, \phi \in D$, but $\phi$ is not a multiplier (see Example 2 following Theorem 1 ).

In the next proposition we require the function classes lip $\alpha, 0<\alpha<1$. These are the analytic functions (necessarily continuous on the closed disc) for which $\lim \mid \phi(z)$ $-\phi(w)|/| z-\left.w\right|^{\alpha}=0$, as $|z-w| \rightarrow 0$. For a discussion of related material see, for example, Duren [11, Chapter 5] or Zygmund [41, Chapter VII, §5]. In particular, $\phi$ is in lip $\alpha$ if and only if $M_{\infty}\left(\phi^{\prime}, r\right)=o\left((1-r)^{\alpha-1}\right)$. (The proof is the same as the corresponding result, due to Hardy and Littlewood, for Lip $\alpha$; see Duren [11, Theorem 5.1].)

Proposition 19. If $\phi$ is holomorphic in $\Delta$ and if

$$
M_{p}\left(\phi^{\prime}, r\right)=\left(\frac{1}{2 \pi} \int_{0}^{2 \pi}\left|\phi^{\prime}\left(r e^{i \theta}\right)\right|^{p} d \theta\right)^{1 / p} \in L^{2}(d r)
$$

for some $p>2$, then:

(a) $\phi \in \operatorname{lip}(1 / 2-1 / p)$,

(b) $\phi^{\prime} \cdot H^{2 p /(p-2)} \subset B$,

(c) $D_{\alpha} \subset H^{2 p /(p-2)}$ for $\alpha>2 / p$,

(d) $\phi \in M(D)$.

RemarK. If $p=2$ then (25) is equivalent to $\phi \in D$. If $p=\infty$ we use the supremum norm in (25); this requires minor changes in the proof below.

Proof. (a) We require the following result: if $h \in L^{1}(0,1)$ is an increasing function, then $h(r)=o\left((1-r)^{-1}\right)$. Indeed, if $\varepsilon>0$ is given then there exists $r_{0}$ such that for $r>r_{0}$ we have

$$
\varepsilon>\int_{r}^{1} h(t) d t \geqslant h(r)(1-r),
$$

and the result follows. Applying this to the function $M_{p}\left(\phi^{\prime}, r\right)^{2}$ we have $M_{p}\left(\phi^{\prime}, r\right)=$ $o\left((1-r)^{-1 / 2}\right)$. By a theorem of Hardy and Littlewood (see Theorem 5.9, p. 84 in [11]) this implies that

$$
M_{\infty}\left(\phi^{\prime}, r\right)=o\left((1-r)^{-1 / 2-1 / p}\right) .
$$

By the Hardy-Littlewood theorem referred to earlier this establishes (a). 
(b) We apply Hölder's inequality with indices $p / 2$ and $\beta=p /(p-2)$ to obtain

$$
\int_{0}^{2 \pi}\left|\left(\phi^{\prime} f\right)\left(r e^{i \theta}\right)\right|^{2} d \theta \leqslant\left(\int\left|\phi^{\prime}\left(r e^{i \theta}\right)\right|^{p}\right)^{2 / p}\left(\int\left|f\left(r e^{i \theta}\right)\right|^{2 \beta}\right)^{(p-2) / p} .
$$

If $f \in H^{2 \beta}$, then by (25) the right side is in $L^{1}(d r)$. Thus $\phi^{\prime} f \in B$, as required.

(c) Let $f=\sum a_{n} z^{n} \in D_{\alpha}$ for $\alpha>2 / p$. Let $q=2 p /(p+2)$; thus $1<q<2$. Thus for the conjugate index we have $q^{\prime}=2 p /(p-2)$. Then by Hölder's inequality (with exponents $2 / q$ and $2 /(2-q)$ ) we have

$$
\begin{aligned}
\sum\left|a_{n}\right|^{q} & =\sum(n+1)^{q \alpha / 2}\left|a_{n}\right|^{q}(n+1)^{-q \alpha / 2} \\
& \leqslant\|f\|_{D_{\alpha}}^{q}\left(\sum(n+1)^{-q \alpha /(2-q)}\right)^{(2-q) / 2} .
\end{aligned}
$$

This is finite since $q \alpha>2-q$. Hence by the Hausdorff-Young theorem (Zygmund [41, Theorem XII.2.3(ii)]) we see that $f \in H^{q^{\prime}}$, as required.

(d) From (a) we have $\phi \in A \subset H^{\infty}$, and from (c) we have $\phi^{\prime} D \subset B$; the result follows from this.

LEMMA 11. Let $\left\{d_{j}\right\}$ be positive real numbers with $d_{j+1} / d_{j}>s>1$ for all $j$. Then

(a) $\sum_{j=1}^{k} d_{j}<s d_{k} /(s-1)(k=1,2, \ldots)$,

(b) $\sum_{j=k+1}^{\infty} d_{j}^{-1}<d_{k}^{-1} /(s-1)(k=1,2, \ldots)$.

Proof. (a) We have $d_{k-i}<s^{-i} d_{k}$. Thus

$$
\sum_{1}^{k} d_{j} \leqslant\left(\sum_{0}^{k-1} s^{-i}\right) d_{k}<\left(\sum_{0}^{\infty} s^{-i}\right) d_{k}=\frac{s}{s-1} d_{k} .
$$

(b) We have $\left(d_{k+i}\right)^{-1}<s^{-i} d_{k}^{-1}$. Thus

$$
\sum_{i=1}^{\infty}\left(d_{k+i}\right)^{-1}<\left(\sum_{1}^{\infty} s^{-i}\right) d_{k}^{-1}=\frac{1}{s-1} d_{k}^{-1}
$$

Proposition 20. If $\phi \in D$ and if $\hat{\phi}(j)=0$ for $j \notin\left\{n_{k}\right\}_{1}^{\infty}$, where $n_{k+1} / n_{k}>q>1$ for all $k$, then:
(a) $M_{\infty}\left(\phi^{\prime}, r\right) \in L^{2}(d r)$,
(b) $\phi \in \operatorname{lip} \frac{1}{2}$,
(c) $\phi^{\prime} H^{2} \subset B$,
(d) $\phi \in M(D)$.

Proof. By Proposition 19, (b), (c) and (d) all follow from (a). To prove (a), let $\phi(z)=\sum_{1}^{\infty} a_{k} z^{n_{k}}$. If $b_{k}=n_{k}^{1 / 2}\left|a_{k}\right|$, then $\left\{b_{k}\right\} \in l^{2}$, since $\phi \in D$. We have

$$
\begin{gathered}
\left|\phi^{\prime}\left(r e^{i \theta}\right)\right| \leqslant \sum_{1}^{\infty} n_{k}\left|a_{k}\right| r^{n_{k}-1}, \\
M_{\infty}\left(\phi^{\prime}, r\right)^{2} \leqslant \sum_{j, k=1}^{\infty} n_{j} n_{k}\left|a_{j} a_{k}\right| r^{n_{j}+n_{k}-2}, \\
\int_{0}^{1} M_{\infty}\left(\phi^{\prime}, r\right)^{2} d r \leqslant \sum_{j, k=1}^{\infty} \beta_{j k} b_{j} b_{k}, \quad \beta_{j k}=\frac{\left(n_{j} n_{k}\right)^{1 / 2}}{n_{j}+n_{k}-1} .
\end{gathered}
$$


(We assume $n_{1} \geqslant 1$.) The proof will be completed if we show that the matrix $\left(\beta_{j k}\right)$ $(j, k=1,2, \ldots)$ represents a bounded operator on $l^{2}$. To do this we apply the "Schur test" in the original form due to Schur: if $\beta_{j k} \geqslant 0$ and if

$$
\sum_{j} \beta_{j k} \leqslant c_{1}, \quad \sum_{k} \beta_{j k} \leqslant c_{2},
$$

then $\left(\beta_{j k}\right)$ is bounded by $\left(c_{1} c_{2}\right)^{1 / 2}$. For a proof and generalization see [42]; for the history of this inequality see [45].

Since our matrix is symmetric: $\beta_{i j}=\beta_{j i}$, we need only verify the first condition in (26). Since $n_{j} \geqslant 1$ we have

$$
\beta_{j k} \leqslant\left(n_{j} n_{k}\right)^{1 / 2} / n_{k}=n_{j}^{1 / 2} n_{k}^{-1 / 2} ;
$$

the inequality obtained by interchanging $j$ and $k$ is also valid. By (27) and Lemma 11(a),

$$
\sum_{j=1}^{k} \beta_{i j} \leqslant n_{k}^{-1 / 2} \sum_{1}^{k} n_{j}^{1 / 2}<\frac{s}{s-1} \quad\left(s=q^{1 / 2}\right) .
$$

Also, by (27) with $j, k$ reversed, and by Lemma 11(b),

$$
\sum_{j=k+1}^{\infty} \beta_{i j} \leqslant n_{k}^{1 / 2} \sum_{k+1}^{\infty} n_{j}^{-1 / 2}<\frac{1}{s-1} \quad\left(s=q^{1 / 2}\right) .
$$

Inequality (26) now follows, with $c_{1}=c_{2}=(s+1) /(s-1)$. This completes the proof.

In conclusion we mention three more problems.

Question 17. If $\phi \in D \cap(\operatorname{Lip} \varepsilon)$ for some $\varepsilon>0$, must $\phi \in M(D)$ ? Suppose $\phi$ is merely Dini continuous?

Question 18. If $f_{1}, \ldots, f_{n} \in H^{\infty} \cap D$ and if $\Sigma\left|f_{i}(z)\right|>c>0$ in $\Delta$, then do there exist $g_{1}, \ldots, g_{n} \in H^{\infty} \cap D$ such that $\sum f_{i} g_{1}=1$ ?

The same question could be posed with $H^{\infty} \cap D$ replaced by $M(D)$, or even by $M(E)$ where $E$ is a general Banach space of analytic functions. In this generality even the case $n=1$ is unknown.

Question 19. If $E$ is a Banach space of analytic functions, if $\phi \in M(E)$, and if $|\phi(z)|>c>0$ in $G$, then is $1 / \phi$ in $M(E)$ ?

This is correct for $M(D)$ (this follows easily since we need only check that $\left(1 / \phi^{\prime}\right)$ multiplies $D$ into $B$ ).

Added in proof. 1. The result of J. Roberts referred to after Proposition 4 will appear in the Illinois Journal of Mathematics.

2. The theorem of Korenblum that was used in the proof of Theorem 4 has been extended to some other Banach algebras of analytic functions. See, for example, A. Matheson, Approximation of analytic functions satisfying a Lipschitz condition, Michigan Math. J. 25 (1978), 289-298; and F. A. Shamoyan, Closed ideals in algebras of analytic functions that are smooth on the boundary, Izv. Akad. Nauk Armyan SSR Ser. Mat. 16 (1981), 173-191. 
3. The condition $\Sigma(n \log n)|\hat{\phi}(n)|^{2} \geqslant \infty$ (Proposition 18(b)), is equivalent to saying that multiplication by $\phi^{\prime}$ is a Hilbert-Schmidt operator from $D$ to $B$.

4. Proposition 19(c) implies that $D \subset H^{r}$ for all $r<\infty$. This can also be obtained by showing that $D \subset V M O A$ (equivalently: if $f \in D$ then there is a continuous function $\phi$ on $\partial \Delta$ such that $\hat{\phi}(n)=\hat{f}(n), n \geqslant 0)$.

5. Korenblum has characterized the cyclic vectors in the space $A^{-\infty}$, that is, the space of analytic functions in $\Delta$, for which

$$
|f(z)| \leqslant a /(1-|z|)^{b}, \quad|z|<1,
$$

for some $a, b>0$ (depending on $f$ ). This space is conjugate to the space $A^{\infty}$. See his paper: A Beurling-type theorem, Acta Math. 138 (1976), 265-293. For further results in this direction see also the papers of N. K. Nikolskii: A criterion for weak invertibility in spaces of analytic functions that are defined by growth conditions, Investigations on Linear Operators and the Theory of Functions. III, Zap. Naučn. Sem. Leningrad Otdel. Mat. Inst. Steklov. (LOMI) 30 (1972), 106-129; Selected problems in weighted approximation and in spectral analysis, Trudy Mat. Inst. Steklov. 120 (1974), 4-271. We mention one further paper in this direction: S. A. Apresjan, Uniqueness theorems and the localization of ideals in algebras of analytic functions with restrictions on the growth, Dokl. Akad. Nauk SSSR 229 (1976), 1033-1036.

\section{BIBLIOGRAPHY}

1. D. Aharonov, H. S. Shapiro and A. L. Shields, Weakly invertible elements in the space of square-summable holomorphic functions, J. London Math. Soc. (2) 9 (1974/5), 183-192.

2. W. B. Arveson, A density theorem for operator algebras, Duke Math. J. 34 (1967), 635-647.

3. S. Banach, Théorie des opérations linéaires, Warszawa-Lwow, 1932.

4. A. Beurling, Ensembles exceptionnels, Acta Math. 72 (1940), 1-13.

5. __ On two problems concerning linear transformations in Hilbert space, Acta Math. 81 (1949), $239-255$

6. J. E. Brennan, Approximation in the mean by polynomials on non-Carathéodory domains, Ark. Mat. 15 (1977), 117-168.

7. L. Carleson, On a class of meromorphic functions and its associated exceptional sets, Appelbergs Boktryckeri, Uppsala, 1950.

8. Sets of uniqueness for functions regular in the unit circle, Acta Math. 87 (1952), 325-345.

9. A A representation formula for the Dirichlet integral, Math. Z. 73 (1960), 190-196.

10. N. Dunford and J. T. Schwartz, Linear operators. I, Interscience, New York, 1958.

11. P. L. Duren, Theory of $H^{p}$ spaces, Pure and Appl. Math., vol. 38, Academic Press, New York, 1970.

12. P. L. Duren, B. W. Romberg and A. L. Shields, Linear functionals on $H^{p}$ spaces with $0<p<1$, J. Reine Angew. Math. 288 (1969), 32-60.

13. J. Garnett, Bounded analytic functions, Pure and Appl. Math., vol. 96, Academic Press, New York, 1981.

14. V. P. Havin, A generalization of the Privalov-Zygmund theorem on the modulus of continuity of conjugate functions, Izv. Akad. Nauk Armyan. SSR 6 (1971), 252-287. (Russian)

15. W. Hayman, On Nevanlinna's second theorem and extensions, Rend. Circ. Mat. Palermo (2) 2 (1953), 346-392.

16. E. Hille and R. S. Phillips, Functional analysis and semigroups, Amer. Math. Soc. Colloq. Publ., vol. 31, Amer. Math. Soc., Providence, R. I., 1957.

17. K. Hoffman, Banach spaces of analytic functions, Prentice-Hall, Englewood Cliffs, N. J., 1962.

18. J.-P. Kahane and R. Salem, Ensembles parfaits et séries trigonométriques, Actualités Sci. Indust., no. 1301, Hermann, Paris, 1963.

19. R. P. Kopp, A subcollection of algebras in a collection of Banach spaces, Pacific J. Math. 30 (1969), 433-435. 
20. B. I. Korenblum, Functions holomorphic in a disc and smooth in its closure, Dokl. Akad. Nauk SSSR 200 (1971), 24-27; English transl., Soviet Math. Dokl. 12 (1971), 1312-1315.

21. __ Invariant subspaces of the shift operator in a weighted Hilbert space, Mat. Sb. 89(131) (1972), 110-137; English transl., Math. USSR Sb. 18 (1972), 111-138.

22. __ Cyclic elements in some spaces of analytic functions, Bull. Amer. Math. Soc. (N.S.) 5 (1981), 317-318.

23. S. N. Mergelyan, On completeness of systems of analytic functions, Uspehi Mat. Nauk 8 (1953), 3-63; English transl., Amer. Math. Soc. Transl. (2) 19 (1962), 109-166.

24. A. Nagel, W. Rudin and J. H. Shapiro, Tangential boundary behavior of functions in Dirichlet-type spaces, Ann. of Math. (2) 116 (1982), 331-360.

25. E. Nordgren, Composition operators on Hilbert spaces, Hilbert Space Operators, Lecture Notes in Math., vol. 693, Springer-Verlag, Berlin, Heidelberg and New York, 1978.

26. R. E. A. C. Paley and A. Zygmund, On some series of functions, 1, 2, Proc. Cambridge Philos. Soc. 26 (1930), 337-357, 458-474.

27. R. C. Roan, Composition operators on the space of functions with $H^{p}$-derivative, Houston J. Math. 4 (1978), 423-438.

28. L. A. Rubel, A. L. Shields and B. A. Taylor, Mergelyan sets and the modulus of continuity of analytic functions, J. Approx. Theory 15 (1975), 23-40.

29. J. V. Ryff, Subordinate $H^{p}$ functions, Duke Math. J. 33 (1966), 347-354.

30. R. Salem and A. Zygmund, Capacity of sets and Fourier series, Trans. Amer. Math. Soc. 59 (1946), 23-41.

31. H. S. Shapiro, Weakly invertible elements in certain function spaces, and generators in $l_{1}$, Michigan Math. J. 11 (1964), 161-165.

32. Some remarks on weighted polynomial approximations of holomorphic functions, Math. Sb. 73(115), (1967), 320-330; English transl., Math. USSR Sb. 2 (1967), 285-294.

33. J. H. Shapiro, Cyclic inner functions in Bergman spaces, preprint (not for publication).

34. A. L. Shields, Weighted shift operators and analytic function theory, Topics in Operator Theory (C M. Pearcy. ed.) Math. Surveys, no. 13, Amer. Math. Soc., Providence, R. I., 1974, pp. 49-128 (second printing, with addendum, 1979).

35. _ Cyclic vectors in some spaces of analytic functions, Proc. Royal Irish. Acad. Sect. A 74 (1974), 293-296.

36. An analogue of a Hardy-Littlewood-Fejer inequality for upper triangular trace class operators, Math. Z. 182 (1983), 473-484.

37. D. A. Stegenga, Multipliers of the Dirichlet space, Illinois J. Math. 24 (1980), 113-139.

38. G. D. Taylor, Multipliers on $D_{\alpha}$, Trans. Amer. Math. Soc. 123 (1966), 229-240.

39. M. Tsuji, Potential theory in modern function theory, Maruzen, Tokyo, 1959.

40. E. T. Whitttaker, and G. N. Watson, A course of modern analysis, 3rd ed., Cambridge Univ. Press, London and New York, 1920.

41. A. Zygmund, Trigonometric series, 2nd ed., Vol. I., Cambridge Univ. Press, London and New York, 1959.

42. Arlen Brown, P. R. Halmos and A. L. Shields, Cesàro operators, Acta Sci. Math. (Szeged) 26 (1965), 125-137.

43. P Hartman and R. Kershner, The structure of monotone functions, Amer. J. Math. 59 (1937), 809-822.

44. H. S. Shapiro, Monotone singular functions of high smoothness, Michigan Math. J. 15 (1968), 265-275.

45. A. L. Shields, An analogue of the Fejer-Riesz theorem for the Dirichlet space (Conf. on Harmonic Analysis in honor of Antoni Zygmund), Vol. II, Wadsworth, Belmont, Calif., 1982, pp. 810-820.

Department of Mathematics, Wayne State University, Detroit, Michigan 48202

Department of Mathematics, University of Michigan, ANN ARbor, Michigan 48109 